\title{
Has Time Come to Switch from Duty-Cycled MAC Protocols to Wake-up Radio for Wireless Sensor Networks?
}

\author{
Joaquim Oller*, Ilker Demirkol*, Jordi Casademont*, Josep Paradells*, Gerd Ulrich Gamm ${ }^{\dagger}$ and Leonhard Reindl ${ }^{\dagger}$ \\ *Department of Telematics Engineering \\ Universitat Politècnica de Catalunya \\ i2CAT Foundation \\ Barcelona, Spain \\ Email: \{joaquim.oller, ilker.demirkol, jordi.casademont, josep.paradells\}@entel.upc.edu \\ ${ }^{\dagger}$ Department of Microsystems Engineering - IMTEK \\ University of Freiburg, Germany \\ Email: \{gerd.ulrich.gamm,reindl\}@imtek.uni-freiburg.de
}

\begin{abstract}
Duty-cycled Medium Access Control (MAC) protocols certainly improve the energy efficiency of wireless networks. However, most of these protocols still suffer from severe degrees of overhearing and idle listening. These two issues prevent optimum energy usage, a crucial aspect in energy-constrained wireless networks such as Wireless Sensor Networks (WSN). Wake-up Radio (WuR) systems drastically reduce these problems by completely switching off the nodes' MicroController Unit (MCU) and main radio transceiver until a secondary, extremely low-power receiver is triggered by a particular wireless transmission, the so called Wake-up Call. Unfortunately, most WuR studies focus on theoretical platforms and/or custom-built simulators. Both these factors reduce the associated usefulness of the obtained results. In this paper, we model and simulate a real, recent and promising WuR hardware platform developed by the authors. The simulation model uses time and energy consumption values obtained in the laboratory and does not rely on custombuild simulation engines but rather on OMNET++ simulator. The performance of the WuR platform is compared with four of the most well-known and widely employed MAC protocols for WSN under three real-world network deployments. The paper demonstrates how the use of our WuR platform presents numerous benefits in several areas, from energy-efficiency and latency to packet delivery ratio and applicability, and provides the essential information for serious consideration of switching duty-cycled MAC-based networks to WuR.
\end{abstract}

Index Terms-Wake-up Radio, energy-efficient networking, OMNET++, WSN.

\section{INTRODUCTION}

$\mathbf{T}$ HE purposes of the many Medium Access Control (MAC) protocols proposed in the literature for Wireless Sensor Networks (WSN) are diverse; while some focus on improving data throughput in bursty traffic conditions, others focus on maximizing energy efficiency. However, regardless of their target, most have the common feature of relying on dutycycling for their implementation; that is, periodical activation

This work is supported in part by the Spanish Government through projects TEC2009-11453, TEC2012-32531 and RYC-2013-13029 grant. J. Oller was supported by FEDER and by the Spanish Government through a FPI grant. followed by a sleep period of the main radio interface of the wireless sensor mote. Unfortunately, while the introduction of such duty-cycling provides important energy benefits over an always-on approach, MAC protocols for WSN still suffer, to a greater or lesser extent, from one or more issues. For example, idle listening occurs when a node listens to the wireless medium during periods when no communication is taking place. Overhearing, in turn, occurs when a node listens to communications intended for another node. In addition, all duty-cycling based approaches also suffer from implicit additional latency, since no information is neither sent or received until the nodes enter their active period. Thus, dutycycling bounds the energy efficiency of MAC protocols, and this in turn affects the network performance. This circumstance has led designers to implement a panoply of different MAC protocols to fit different application requirements, resulting in what is known as the MAC Alphabet soup for WSN [1].

Recently, Wake-up Radio (WuR) systems have constituted a good alternative for tackling the issues to which duty-cycled MAC protocols are prone. In WuR, an additional, simplistic, hardware receiver, the so-called Wake-up Receiver (WuRx), is attached to an interrupt-capable General Purpose Input Output (GPIO) pin of the MicroController Unit (MCU) of the sensor node. This MCU is configured to remain in its lowest power mode by default. The purpose of the WuRx is to detect a Wake-up Call (WuC) sent by a Wake-up Transmitter (WuTx) in the remote nodes prior to any wireless data communication. Upon such WuC detection, the WuRx generates an interrupt to the node's MCU to which it is attached, and causes it to switch from sleep to active mode. Next, the MCU activates the main wireless radio interface and the participating nodes may proceed to communicate in a traditional fashion.

Because of this on-demand paradigm of $\mathrm{WuR}$, where nodes only operate when their intervention is really required, idle listening, overhearing and latency issues are drastically reduced. At the same time, since WuRx designs operate in the $\mu \mathrm{A}$ order, the nodes' current consumption is reduced by a factor of 1000 , 
a crucial factor for devices such as wireless sensors that are usually powered by means of small batteries. However, such low current consumption comes at the cost of worse receiver sensitivity, which in turn implies that the WuTx has to transmit at a higher power.

As the number of MAC and WuR proposals grows in the literature, there are many unknowns that remain unaddressed, among the most important being the different performance they feature when applied to realistic applications among the most important ones. In addition, since some designs are proposed entirely by simulation, their implementation and performance evaluations rely on custom-designed software solutions, a factor which limits the reproducibility of the obtained results. In this paper, we contemplate a real WuR design and compare its performance to four widely known MAC protocols. We describe and model our platform in OMNET++ [2] and MiXiM [3] to avoid custom-built simulation software. OMNET++ provides reliable primitives for wireless signal propagation, energy consumption, etc., as well as the means to extract detailed simulation results. We additionally employ the framework MiXiM on top of OMNET++ because of its focus on WSN purposes. Compared to OMNET++, MiXiM provides primitives specific to wireless sensor, body area and ad-hoc networks. These include characterizations of known hardware radio transceivers such as $\mathrm{CC} 2420$ or CC1101, as well as implementations of two largely recognized MAC protocols for WSN; that is, B-MAC and unslotted IEEE 802.15.4. For greater significance of the results, we develop and add two implementations of broadly known MAC protocols to MiXiM, $\mathrm{X}-\mathrm{MAC}$ [4] and RI-MAC [5], by strictly following the design guidelines in their respective research papers, altogether with our above-mentioned simulation model for our real hardware WuR platform, introduced in [6] and characterized in [7]. For the WuR parameter characterization, we employ empirical time and current consumption values measured from real evaluation boards. We analyze the performance of the five approaches under three scenarios derived from real-life WSN use cases for numerous metrics such as power and energy consumption, battery lifetime, latency and Packet Delivery Ratio (PDR). Both individual node and global network contexts are considered.

We strongly consider the on-demand nature and the energy savings provided by WuR as a decisive factor for rethinking applications from using traditional MAC protocols. However, and as it is logical, such a switching obviously has to occur with minimum overhead. Thus, in this paper we concentrate on providing the necessary data to evaluate the feasibility of such a change for different kinds of applications. The simulations performed contemplate either single-hop networks or static and mobile multi-hop networks. Practically any WSN falls within one of these categories. Hence, the results in this paper reflect the performance offered by the five different communication methods for scenarios that are commonly found in the literature. To the best of the authors' knowledge, because of the broad range of scenarios considered and the use of values from real WuR hardware, this paper is among the first of its nature, if not the first. The results obtained, altogether with the knowledge basis given therein, are aimed at demonstrating to the network designers about the feasibility, benefits and convenience of deploying WSN featuring WuR rather than traditional MAC protocols.

The reminder of the paper is structured as follows. In Section II, we introduce the state of the art of WuR simulation and its comparison to traditional MAC protocols. Next, in Section III we describe the complete employed simulation framework and the MAC protocols and WuR implementations in OMNET++ and MiXiM. The proposed scenarios under evaluation are presented in Section IV, together with a complete analysis of the performance in terms of numerous metrics for each approach under each of them. Finally, Section V concludes the paper.

\section{RELATED WORK}

$\mathrm{WuR}$ is a recent and emerging research topic. Accordingly, numerous WuR designs have appeared during recent years. For a detailed state of the art on WuR studies, as well as a complete overview of their basic operation, we refer the reader to [7]. In fact, the number of studies comparing WuR performance to that achieved by WSN MAC protocols is quite limited and their application areas are very specific. Instead, a few studies, e.g. [8], focus mainly on comparisons among WuR. However, such studies overlook important or novel WuR functions such as multi-hop WuR communications, and do not provide any information related to the different performance provided by WuR when compared to traditional MAC approaches. In addition, the simulation software employed is often not clearly described. We consider that any analysis on this research area should clearly address each one of these aspects.

In [9], a comparison is performed between two WuRx designs requiring $50 \mu \mathrm{W}$ and traditional preamble-sampling MAC protocols. Simulations are performed in MATLAB but node placements are random, thus the results in the paper are difficult to extrapolate to real-world applications such as mobile data-collectors and/or planned node deployments. Furthermore, the WuRx designs in [9] require about 5 times more power than that presented and evaluated in this paper, and unfortunately do not provide any data related to the range achieved by the WuR operation. In fact, the work in [9] only focuses on analytical aspects, such as the required number of nodes to achieve full connectivity in an application area via multi-hop capabilities.

The authors in [10] perform a comparison between simulation and an ideal mathematical analysis of wireless nodes equipped with a real WuR system featuring a power consumption of $125 \mu \mathrm{W}$ for the WuRx. To this end, the WuR design is modeled and the measurements obtained used as input in the simulation for more significant results. The authors investigate the effect of the number of hops on the network's packet delivery ratio and effective communication range. As in the previous case, nodes are randomly distributed. The paper does not contemplate other important aspects such as latency measurements, detailed energy distribution for the participating nodes or the effect of interferer nodes, among others. Instead, and in a similar manner to [8], the main purpose of the paper is to determine the coverage of WuR 
systems when compared to traditional approaches. While this subject is important because of the shorter range of WuR compared to traditional transceivers, a complete application analysis should consider other concurrent aspects. In addition, the WuR system analyzed in [10] requires up to 15 times more power than our WuR system. This drastically reduces the network lifetime, an evaluation which is omitted in the paper.

A complete simulation for a binary-tree scenario comparing B-MAC, IEEE 802.15.4 and a WuR design called RFIDImpulse is presented in [11]. While this work considers a realworld application and relevant MAC protocols, the design of the WuR is not detailed, nor the operational range information is provided. As a result, the reproducibility and applicability of the results obtained in [11] are limited. The authors address this issue in [12], which provides complete information about a WuRx design based on a commercial active RFID tag. More specifically, the work in [12] analyzes the performance of TelosB and MicaZ sensor platforms when equipped with such WuRx in order to decide the lowest power mode that their corresponding MSP430 and Atmega128 MCU can work at different application data rates. However, the WuTx includes an RFID reader, the power consumption of which limits this design's applicability to multi-hop scenarios.

In this paper, we first intend to provide a lean and modular OMNET++ simulation model for WuR systems. This simulation model represents a real hardware platform completely developed from scratch, the details of which can be found in [6], [7]. In our preliminary work in [13] consisting a single scenario, we evaluated a single performance metric and two default WSN MAC protocols from MiXiM without any interference or mobility conditions. However, in this paper we contemplate three realistic application scenarios, four WSN MAC protocols and two WuR flavors, or variants. The simulator implementation of the Wake-up Radio module has also been redesigned to provide a more flexible design to be able host any WuR implementation.

In this paper, we completely describe these two flavors in full, as well as providing the guidelines to allow designers to properly choose one of these flavors depending on the target application. To provide a fair comparison, the different MAC protocols and WuR implementations in this paper are fed by parameters extracted from real hardware characterizations. To the best of the authors' knowledge, this paper is the first to provide such an extensive, applicable and detailed performance evaluations and comparative analysis in this research area.

\section{Simulation FRAMEWORK FOR MAC PROTOCOLS AND WAKE-UP RADIO}

We have developed our WuR model on MiXiM because of 1) its integration to OMNET++ and 2) the models it provides explicitly for WSN, such as characterizations of common radio transceivers and implementations of B-MAC and IEEE 802.15.4 protocols. Our work is compatible with the latest software versions of OMNET++ and MiXiM 4.3 and 2.3, respectively. In order to achieve an architecture as generic as possible, we consider the node model depicted in Fig. 1, which sits on top of the standard model provided by OMNET++. Since MiXiM focuses on Wireless Sensor Networks, its main contributions take place in the Radio modules.

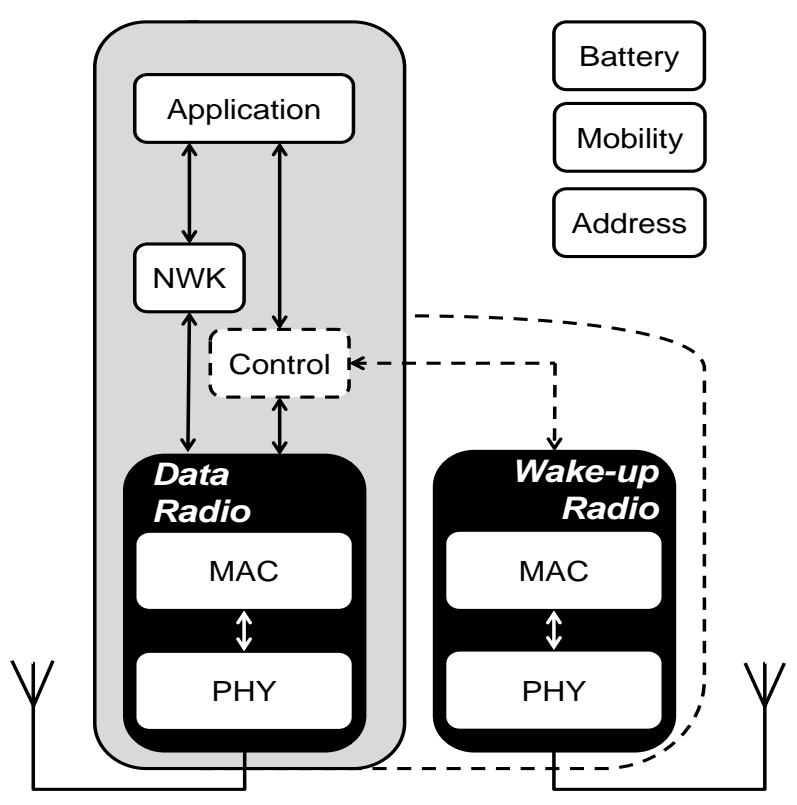

Fig. 1: Node model proposed in OMNET++. MiXiM MAC protocols for WSN are implemented in the Data Radio module. Wake-up Radio addenda are indicated by dotted lines.

In Fig. 1, the Application module contains the code for the user application (e.g., to initialize the node), to generate packets or to perform mobility-aware tasks, such as controlling the node speed in case it is mobile. Next, different routing protocols may be implemented in the underlying Network (NWK) layer module, which may also be omitted. To focus our evaluations on MAC protocols and WuR performances and to achieve fairness, the only changes among different simulation setups take place at the lower radio levels. In other words, the MiXiM's configuration files for B-MAC and XMAC are the same and only differ in the parameter indicating the MAC protocol under analysis. When evaluating WuR, the Wake-up Radio module is enabled. Thus, as shown in Fig. 1, we respect the MiXiM's basic node model and just add the required software modules for WuR designs. These additional Wake-up Radio modules are simply omitted in simulations involving nodes implementing traditional MAC protocols. In other words, the Data radio is the only radio module active in simulated scenarios with nodes implementing the MAC protocols analyzed in this paper; that is, B-MAC, X-MAC, RI-MAC or IEEE 802.15.4. Instead, when evaluating WuR nodes, the main radio is managed by a Transceiver Controller module, depicted as Control block in Fig. 1, which allows the application to monitor and control the status of the transceiver, as is done in WuR platforms. The Battery, Mobility and Address blocks in Fig. 1 provide primitives for averaging the power consumption, setting the node mobility pattern and the address scheme featured by the network, respectively.

In order to evaluate the performance of the nodes in different 
applications, we set the parameters of Application, Mobility and Data Radio modules of nodes accordingly. Thus, our simulation model allows for a panoply of combinations to be tested, which can be done in a plug-and-play manner.

\section{A. Implemented MAC Protocols}

The working principles of different MAC protocols simulated in this paper are depicted in Fig. 2. The first three approaches, i.e., IEEE 802.15.4, B-MAC and X-MAC, represent examples of Transmitter Initiated (TI) protocols. On the other hand, RI-MAC is the reference Receiver Initiated (RI) protocol evaluated in this study. This terminology classifies the protocols depending on which node starts the data communication procedure. Regarding WuR, and because of the flexibility of our WuR model, it is possible to enable any of the two approaches by simply changing a few lines of code. Hence, we include both WuR variants, or flavors, in the analysis in this paper; namely TI-WuR and RI-WuR. In TI-WuR, a node first sends a WuC to wake up the remote node and then sends a data frame afterwards. In RI-WuR, a node wakes up a remote node in order to receive back a data frame, if available. In other words, in TI-WuR the node sending the $\mathrm{WuC}$ and the data frame is the same one. On the other hand, in RI-WuR, the node originally sending the $\mathrm{WuC}$ immediately switches its main radio to receiving mode in order to listen for an incoming data frame from the remote node.
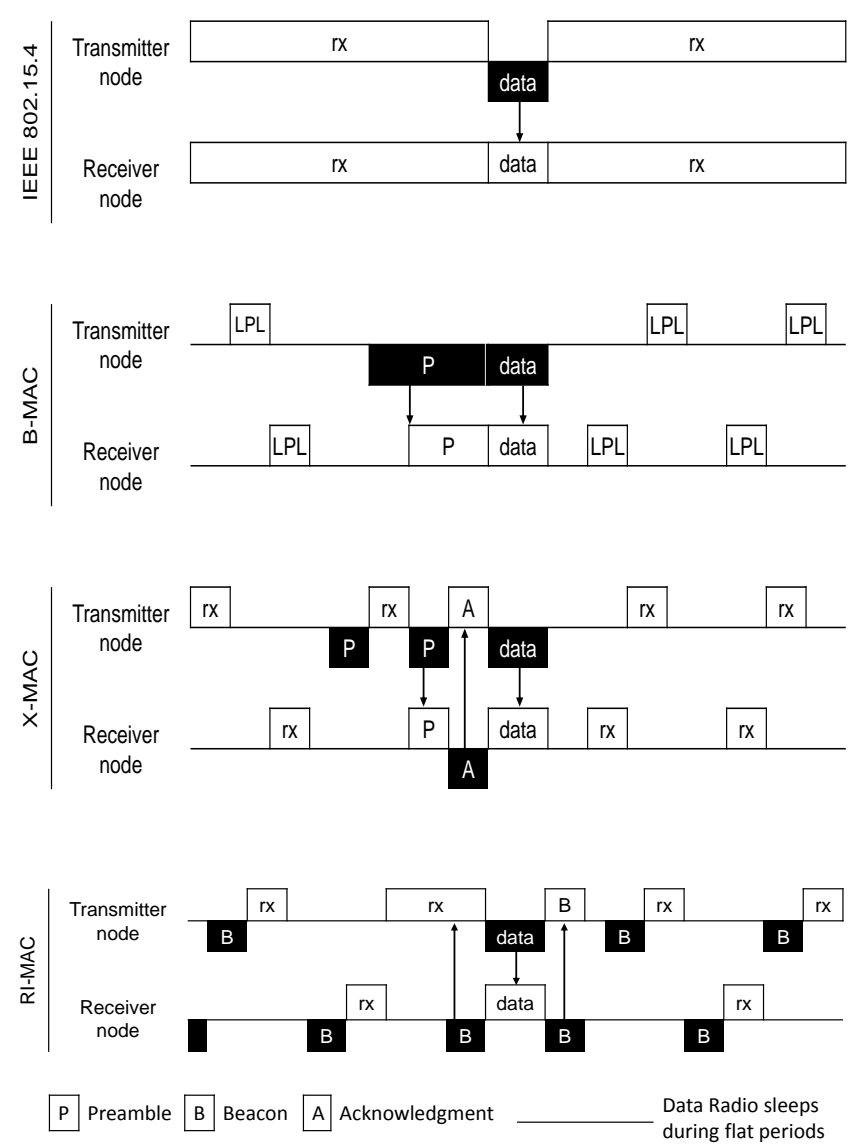

Fig. 2: Working principles of evaluated MAC protocols.
In our evaluations, the Data Radio module in Fig. 1 contains an implementation of one of the following MAC protocols: unslotted IEEE 802.15.4, B-MAC, X-MAC and RI-MAC. We choose these protocols due to several reasons that are enumerated next.

IEEE 802.15.4 is the most commonly employed protocol in WSN nodes, e.g. by ZigBee [14] and 6LoWPAN [15] devices. This standard specifies two different flavors for a) networks comprising a controller node, which coordinates the other nodes by means of beacon frames, and b) for networks without such a controller. The beacon-enabled mode implements a Contention Free Period (CFP) during which the nodes access the medium by using Time Division Multiple Access (TDMA), since the controller manages the scheduling. Thus, in this period, nodes can be duty-cycled. However, the beaconless mode is more common for peer to peer networks, since it does not require and/or depend on a special controller node. In the beaconless mode, nodes have to either be 'always awake', or use some synchronization mechanism (which is beyond the scope of IEEE 802.15.4) for duty-cycling. Since, as shown in Fig. 2, no duty-cycling is performed in beaconless IEEE 802.15.4, this protocol plainly sets a benchmark value in several aspects like data throughput and latency, at the cost of presenting the highest energy consumption of them all. As a Carrier Sense Multiple Access (CSMA)-based approach, in a beaconless IEEE 802.15.4 network a node desiring to access the channel must first assure that no other transmitter is using the medium by means of Clear Channel Assessment (CCA). If the node senses that the medium is busy after CCA operation, it performs a randomized back-off and tries to transmit after the chosen back-off time. Since in CSMAbased protocols, such as in IEEE 802.15.4, no preamble or wake-up packet is needed, transmissions can be performed more quickly than in preamble-based MAC approaches such as B-MAC and X-MAC, which are introduced next. In our performance evaluations, we evaluate the beaconless version of IEEE 802.15.4 coming by default in MiXiM, the performance of which establishes a common base reference for all the obtained results. In the rest of the paper, we refer to this implementation simply as IEEE 802.15.4.

B-MAC [16], also included in MiXiM by default, is a widely known WSN MAC protocol, since it is the default MAC layer for several operating systems for WSN nodes such as for TinyOS [17]. In B-MAC, as shown in Fig. 2, a transmitting node first emits a preamble which is slightly longer than the entire sleeping period of duty-cycling nodes. This timing ensures that the receiver node is able to detect the preamble after the sleeping period of its duty cycle. For such detection, and in order to save energy, the receiver's transceiver is not operated at full power, but it simply performs CCA to detect the presence of a radio transmission. Thus, the preamble is detected by a Low Power Listening (LPL) strategy using a simple channel energy detection method) by the receiver, which waits until its end and then switches to real data reception mode, where the transceiver presents higher current consumption since from this point on it requires demodulation and decoding capabilities. This preamble-based approach of B-MAC implies severe medium occupancy levels and latency issues. These 
drawbacks can be reduced by increasing the duty-cycle ratio, i.e., by shortening the sleep period of the MAC protocol, which in turn increases the energy consumption. Note that because of its preamble, which is implemented as a constant and uninterrupted flow of bits, B-MAC is executed by byte-level radios such as the $\mathrm{CC} 1000$, where the minimum transmission unit is not packet but byte. This implies that data packets need to be decomposed to bytes and reassembled from the received bytes. Nowadays, this popular radio transceiver has been replaced by the CC1101, which implements both bytelevel and packet-level features.

X-MAC [4] shifts the operation of B-MAC to packet-level radios in order to solve the aforementioned problems. Its performance is known to be better than that of B-MAC, thus it is an imperative protocol to evaluate in our simulations and omnipresent in the related literature. In fact, a variant of XMAC, the so-called X-MAC-UPMA, is the base MAC protocol of Contiki operating system for WSN [18]. Thus, evaluations of X-MAC in this paper can be extrapolated to several similar protocols. In X-MAC, as shown in Fig. 2, the preamble is sliced or strobed, which means that the transmitter alternatively sends short preamble packets and listens to the channel. Unlike B-MAC, such short packets include the address of the intended receiver of the communication. The surrounding nodes that are not addressed can therefore return immediately to sleep in order to reduce overhearing as soon as they detect that the ongoing communication is not intended for them. In turn, the intended node must respond with an acknowledgment (ACK) frame. This behavior solves the long preamble issue in B-MAC and allows for a fairer channel usage. Once the transmitter receives back the ACK, it can proceed to send the data frame. Note that if no ACK is received back, the XMAC preamble may be as long as in B-MAC. X-MAC can be implemented in packet-level radios such as the CC2420, CC2520 or CC1101, the models of which are provided by MiXiM. For our X-MAC implementation, we strictly follow both X-MAC paper [4] and MiXiM design guidelines given in [3].

In certain user applications, it is advisable for the receiver node to start the communication. This paradigm is called Receiver Initiated communication. RI-MAC [5] is the reference WSN MAC protocol for RI communications and presents a noticeably different performance for certain applications when compared to IEEE 802.15.4, B-MAC and X-MAC, which are all Transmitter Initiated protocols. In this paper we include RIMAC in our evaluations in order to broaden the applicability of the results in this paper we include RI-MAC in our evaluations. As shown in Fig. 2, in the active part of its duty-cycle, a node running RI-MAC without any packet to transmit indicates this condition by sending a beacon. Nodes that require the delivery of a data frame to this node proceed to listen to the medium for a prolonged time slot. The reception of the beacon from the ready-to-receive node acts as the trigger to start communication. This procedure can be effectively regarded as the reverse equivalent of a preamble. Upon reception of a beacon from the intended receiver, the transmitter node may proceed to send the data frame. Thus, RI-MAC achieves lower power consumption if the power for packet reception is higher than for transmission and the data traffic and node density is not high, since all the nodes without queued packets contend for sending their beacons during their active period. In addition, RI-MAC does not suffer from long preambles occupying the medium. However, as in B-MAC and X-MAC, it also suffers from an unavoidable constant current consumption because of its periodic beacon sending.

\section{B. Wake-up Radio Design Implementation}

As seen in Fig. 1, WuR nodes in our model feature two radio transceivers; the main transceiver and the wake-up transceiver. This two-radio model enables the simulation of any kind of WuR system, either with two separate radio interfaces or with a shared transceiver. As regards its implementation, the operation of WuR in both Transmitter and Receiver Initiated flavors is shown in Fig. 3.

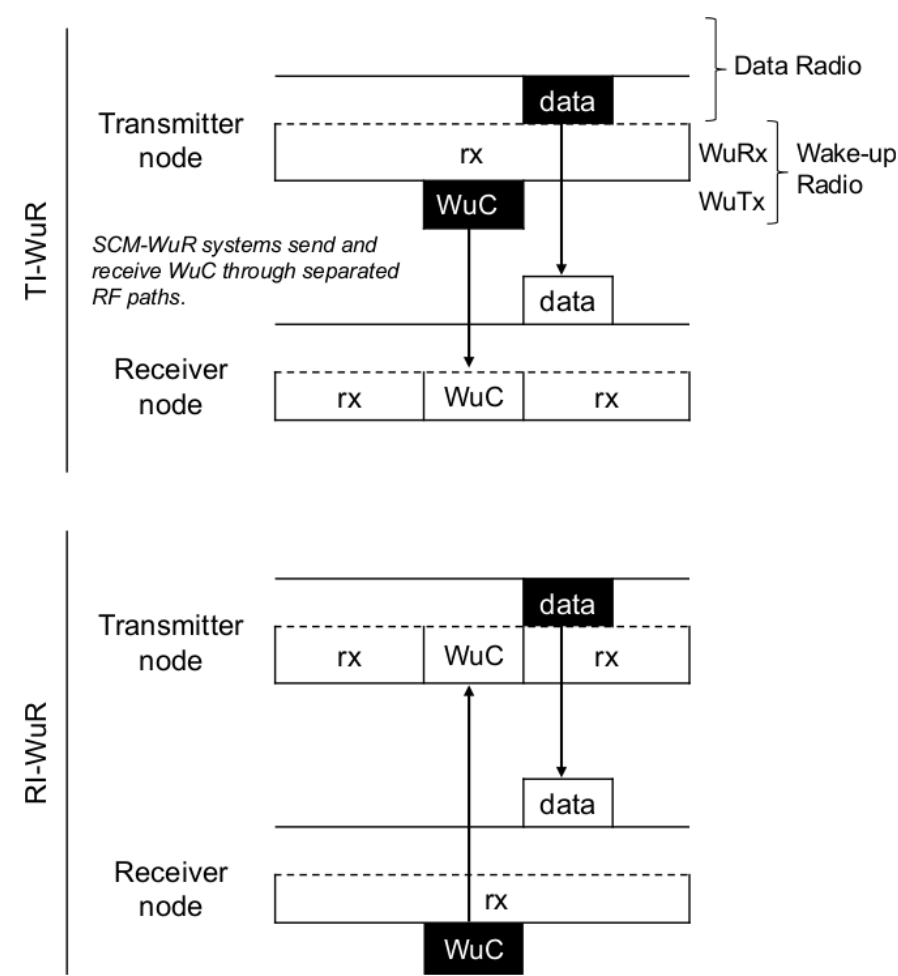

Fig. 3: Working principles of WuR approach. WuRx requires few $\mu \mathrm{A}$ to operate.

In the general case of WuR, MCU and the main data communication transceiver are initially switched off to reduce the energy consumption while the WuRx remains activated to monitor the channel. However, unlike traditional transceivers, WuRx only require very few $\mu \mathrm{A}$ for this purpose. In a WuR system, when a node wants to communicate, it first transmits a $\mathrm{WuC}$ via its WuTx. At the receiver node, a WuRx receiving a $\mathrm{WuC}$ generates an interrupt to wake up the node's MCU, which in turn switches on the main transceiver so that upcoming data frames can be received in a traditional fashion through the main radio. After the MCU of the node is activated, it may perform several tasks before disabling the data transceiver and going to low-power WuR mode again, such tasks being the reception of an incoming data frame (TI-WuR approach), or 
obtaining a measurement from a sensor and sending back the data (RI-WuR approach). In our MiXiM model shown in Fig. 1 , incoming and outgoing WuCs are managed by the Wake-up Radio block, while traditional communications are performed through the Data Radio block employing any MAC protocol. In the evaluations in this paper, the Data Radio block of the WuR-equipped nodes employs IEEE 802.15.4 when active. This Data Radio block is only activated by Control block upon detection of a valid $\mathrm{WuC}$.

In lieu of the additional hardware required by WuRx, employing WuR allows significant simplifications of the MAC protocol for the main radio, as it enables rendezvous-less, asynchronous, on-demand communication. On detecting a valid WuC, a WuRx simply provides an Interrupt ReQuest (IRQ) to the MCU. This can be managed analogously by the MCU as when a button is pressed, and effectively allows all the code required for precise timing in MAC protocols' implementations to be eliminated. On the other hand, while WuR introduce some delay due to the $\mathrm{WuC}$ transmission, this delay is never as high as the preambles used in duty-cycled MAC protocols.

The WuR platform analyzed in this paper is called SubCarrier Modulation Wake-up Radio (SCM-WuR) and its details can be found in [6], [7]. In SCM-WuR, both WuC and data transmissions are generated by the same CC1101 transceiver [19] by dynamically changing the radio settings. The input / output RF path is managed through an antenna switch by the node's MSP430 low-power MCU. SCM-WuR nodes are based on a Low Frequency (LF) $125 \mathrm{kHz}$ integrated circuit, AS3932, which is triggered upon detection of the proper node address in the $\mathrm{WuC}$. In order to decode this address, this circuit features a hardware address correlator that only requires very few $\mu \mathrm{A}$ for its operation. At the WuTx side, to switch from magnetic coupling $(125 \mathrm{kHz})$ to electric coupling $(868 \mathrm{MHz})$, and to achieve longer operational ranges, the $\mathrm{WuC}$ is modulated in an On-Off Keying (OOK) manner by shaping on a time basis a continuous flow of 0xAA bytes, or 0b10101010, at $250 \mathrm{kbps}$, which results in a pseudo- $125 \mathrm{kHz}$ carrier.

By featuring a programmable MCU, a SCM-WuR node may operate as either WuRx or WuTx. This flexibility also becomes very useful when the same node must be able to use both Transmitter and Receiver Initiated approaches. For example, some nodes may be interested in reporting notifications (e.g. sending sensor measurements without a previous query) or may provide responses to queries (e.g. about the last temperature measurement).

Currently, there exist two hardware versions of the SCMWuR platform. In this paper, we consider the one that can operate up to 100 meters [20]. This distance value is among the best in the WuR literature and the first one for which real multi-hop capabilities have been demonstrated [7]. Numerically, a SCM-WuR board features as low as $3.5 \mu \mathrm{A}$ when operating as WuRx in low-power wake-up mode and no WuC is present. This value increases up to $8 \mu \mathrm{A}$ when the $\mathrm{WuRx}$ is decoding the address embedded in an incoming WuC. In regard to the transmitter side, the $\mathrm{WuTx}$ role requires up to $152 \mathrm{~mA}$ when sending a $\mathrm{WuC}$ in order to achieve the 100 meters range. This way, SCM-WuR transmissions thus present operational ranges comparable to traditional wireless sensor communications. However, due to the fact that $\mathrm{WuRx}$ designs are kept simplistic in order to operate in the $\mathrm{mA}$ order of magnitude, $\mathrm{WuC}$ transmissions in SCM-WuR require noticeably more power than conventional data frames to be detected. As a counterpart, and unlike duty-cycling systems, this energy for transmitting a WuC is only employed when really required, instead of periodically checking the wireless medium.

\section{Performance Results}

In this section, we consider three application scenarios which include a single-hop scenario and two multi-hop scenarios, one with static topology and one with mobile topology. We evaluate each of the four WSN MAC protocols and SCMWuR approach under these application scenarios.

\section{A. Evaluated Scenarios}

The three application scenarios are depicted in Fig. 4. Network nodes are colored lighter, while darker ones represent interference sources modeled as contention generators that generate their own transmissions that are not intended for the network under analysis. Interferer nodes run the same MAC protocol as network nodes. However, their transmissions are directed to a node address that is not present in the network evaluated. Thus, they can be considered as collocated networks deployed close to the one under evaluation. For each scenario, we study the effect of diverse metrics such as variable datarates, the speed of mobile node or the duty-cycle featured by nodes implementing the MAC protocols, where appropriate.

The first scenario in Fig. 4a depicts a single-hop use case where a mobile data-collector, e.g., a bus, train, drone or robot, collects information from sensors deployed along its route. The collector node's mobility model is indicated by arrows in the figure and is periodic. The wireless sensors may be attached to trees, garbage containers, or they may also represent energy utility meters installed inside nearby buildings. This simulation corresponds to an ongoing real project in Sant Vicenç dels Horts, Barcelona [21]. Because of the sporadic, single-hop communication nature of this scenario, the bus speed does not affect the investigated system's performance metrics.

The scenario in Fig. 4b depicts a WSN deployed as a multi-hop static binary tree topology, which is common in the WSN literature [11]. Basically, intermediate nodes are in charge of forwarding packets from their immediate child nodes, and these in turn do so from their own descendant nodes. This network configuration is widely employed for monitoring applications, e.g., for precision agriculture.

Finally, the last scenario in Fig. 4c depicts a multi-hop mobile scenario where a mobile data-collector, e.g., a bus, train, drone or robot, collects information from chains of sensors. For example, sensor nodes can be attached to the pillars of a bridge or along the side streets of a main road. Thus, this scenario can be considered as a combination of the previous two. Since, unlike the first scenario, this is a multi-hop scenario, the vehicle speed affects the network performance. A variation of this use case is currently being 


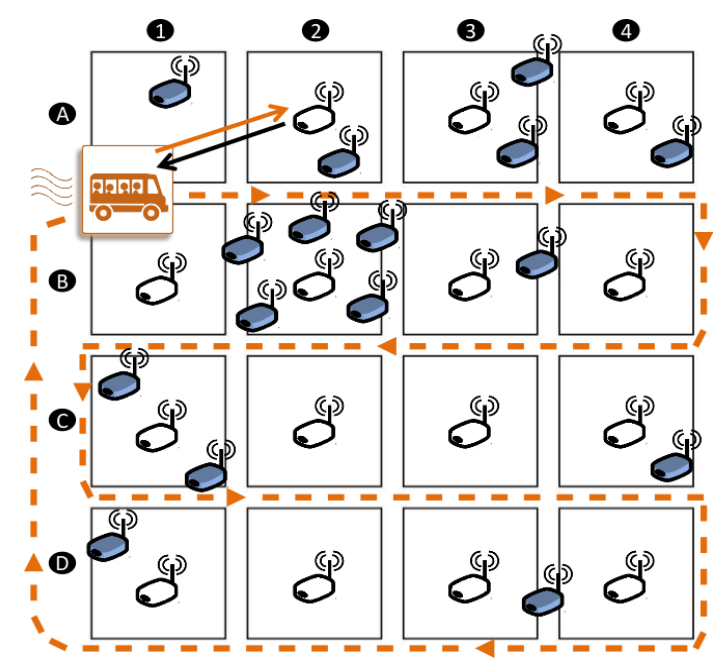

(a)

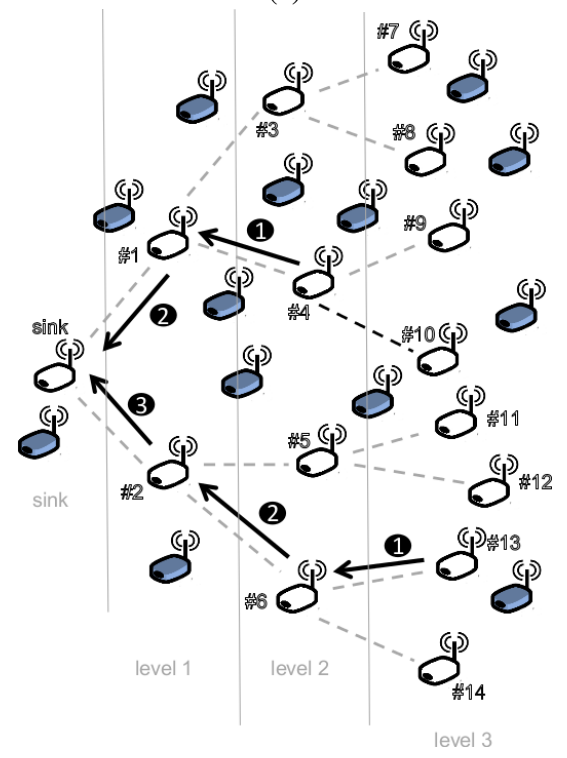

(b)

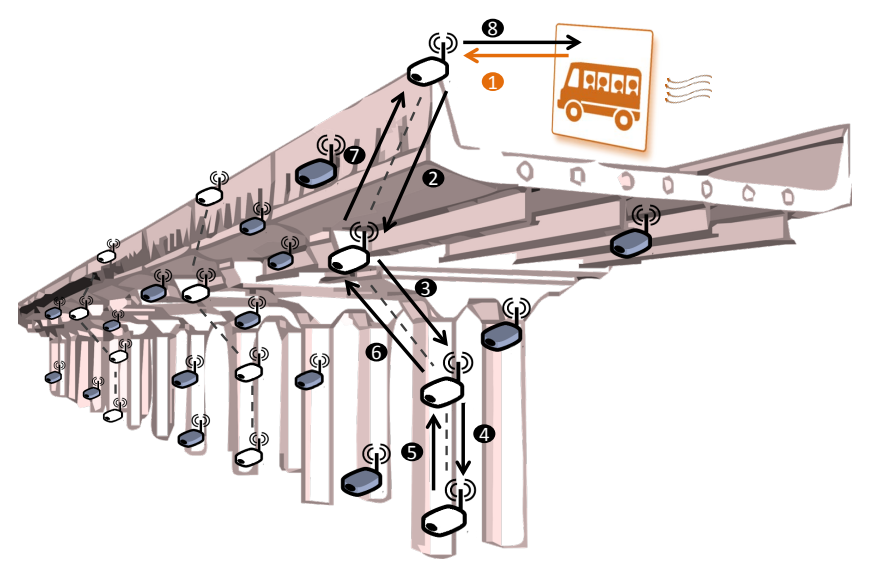

(c)

Fig. 4: Scenarios analyzed in this paper: (a) data-collector mobile single-hop, (b) converge-cast tree or static multi-hop, (c) data-collector mobile multi-hop.
TABLE I: Simulation Parameter Set

\begin{tabular}{|c|c|c|c|}
\hline Approach & Parameter & Value & Unit \\
\hline \multirow{15}{*}{ Common } & Supply Voltage & 3 & $\mathrm{~V}$ \\
\hline & Battery Capacity & 1500 & mAh \\
\hline & Reception Current & 18.8 & $\mathrm{~mA}$ \\
\hline & Transmission Current & 17.4 & $\mathrm{~mA}$ \\
\hline & Sleep Current & 0.02 & $\mu \mathrm{A}$ \\
\hline & Packet Payload & 100 & bytes \\
\hline & Queue Buffer Length & 10 & packets \\
\hline & Maximum Transmission Attempts & 2 & retries \\
\hline & Distance between Nodes & 150 & $\mathrm{~m}$ \\
\hline & Scenario Duration $\mathrm{w} /$ Interferers & 3 & hours \\
\hline & Scenario Duration w/o Interferers & 12 & hours \\
\hline & Default Duty-cycle & 1 & $\%$ \\
\hline & Bit Error Rate & $10^{-8}$ & $\%$ \\
\hline & Signal Model & \multirow{2}{*}{\multicolumn{2}{|c|}{$\begin{array}{c}\text { SimplePathLoss } \\
3.2\end{array}$}} \\
\hline & Path Loss Exponent & & \\
\hline \multirow{3}{*}{ IEEE 802.15 .4} & Bit-rate & 250 & kbps \\
\hline & Slot Duration & 100 & $\mathrm{~ms}$ \\
\hline & Backoff Exponent & 3 to 8 & slots \\
\hline \multirow{2}{*}{ B-MAC } & Bit-rate & 15360 & kbps \\
\hline & Slot Duration & 1 & $\mathrm{~s}$ \\
\hline \multirow{2}{*}{$\mathrm{X}$-MAC } & Bit-rate & 250 & kbps \\
\hline & Slot Duration & 1 & $\mathrm{~s}$ \\
\hline \multirow{2}{*}{ RI-MAC } & Bit-rate & 250 & kbps \\
\hline & Slot Duration & 1 & $\mathrm{~s}$ \\
\hline \multirow{5}{*}{ Wake-up Radio } & Sleep Current & 3.5 & $\mu \mathrm{A}$ \\
\hline & Bit-rate & 250 & kbps \\
\hline & WuC Duration & 12.2 & $\mathrm{~ms}$ \\
\hline & Reception Current (WuRx) & 8 & $\mu \mathrm{A}$ \\
\hline & Transmission Current (WuTx) & 152 & $\mathrm{~mA}$ \\
\hline
\end{tabular}

deployed and monitored in Germany as part of the IB-ISEB project [22]. The bus, tree and bridge applications in Fig. 4 may be considered respectively as networks that require an instantaneous response, a network with stable and constant throughput and a mix between the two.

Table I summarizes the crucial simulation parameters. $\mathrm{Pa}$ rameters for B-MAC and IEEE 802.15.4 are taken from MiXiM implementations. Only the current consumption of the B-MAC transceiver has been explicitly modified and set to be equal to that used for the remaining MAC protocols in order to provide a fair comparison. The parameters of the implementations of X-MAC, RI-MAC and WuR are taken from the respective reference papers [4], [5] and [7].

In order to maximize the representativeness of the results, we perform 10 simulation runs with different random seeds for each parameter combination. Each parameter combination comprises the scenario, communication protocol and metric under analysis. Random seeds are used for different aspects, such as the initial placement of interfering nodes or time offset for periodic packet generation. The obtained results are processed by using specific OMNET++ packages for the R statistics software. We include the $95 \%$ confidence intervals in the plots representing all these repetitions, providing they are scientifically significant.

\section{B. Single-hop Scenario}

The single-hop scenario, shown in Fig. 4a, depicts the use case of a data-collector node which collects information from 15 sensors deployed along its route. In this simulation scenario, the simulation area is about $1 \mathrm{~km}^{2}$ and the vehicle moves at $10 \mathrm{~m} / \mathrm{s}$. A single-hop communication as considered in this scenario is not affected by the vehicle's speed. 
Only statistics for the nodes of the evaluated network (white colored nodes) are collected. Darker interferer nodes are considered to be part of coexisting systems whose transmissions interfere with the system's network under evaluation, and their placement is randomized for each simulation. This single-hop scenario is a very typical case in which the data-collector, or the node which starts the communication, has no power restrictions. On the other hand, the field-deployed nodes must save as much energy as possible by exploiting the infrequent nature of the data communications. It is for this reason that the use of inefficient medium listening procedures must be minimized. This is therefore a clear example of a Receiver Initiated scenario where the receiver is the data collector. For the WuR approach, RI-WuR variant is considered, since the mobile node is the one both sending the $\mathrm{WuC}$ as well as receiving back the data frame. In this section, we analyze the effects of both the presence of interferer nodes and the duty cycle ratio of the nodes implementing MAC protocols.

The data-collector queries nodes by means of their address and expects a response back. In real applications, the datacollector is equipped with a GPS application which determines the sensors to be queried in the area. If such a response fails to arrive in time, the data-collector tries up to the maximum packet retransmission attempts, given in Table I, before removing the query from the queue and proceeding to consult the next node in the grid as it approaches it. For this scenario, we define the PDR as the ratio of responses received back at the mobile node, over the number of performed queries. Thus, the PDR metric represents in a very direct manner the global success of the evaluated communication protocol. However, since nodes in this scenario are queried sporadically, also the energy behavior when the nodes are idle acquires great importance and is therefore also evaluated.

1) Effect of the Duty-Cycle Ratio: In order to quantify the effect of the duty-cycle in the performance of the MAC protocols, we set up a configuration with 30 interferer nodes and vary the ratio of time a node is active, i.e, vary the duty-cycle ratio. The effect of these variations on different metrics can be observed in Fig. 5. In Fig. 5b, the performance bars of SCM-WuR are not visible, since the average power consumption $(30 \mu \mathrm{W})$ is very close to 0 .

As shown in Fig. 5a, the only approaches that the change in the duty-cycle ratio affects significantly are Transmitter Initiated MACs, i.e., B-MAC and X-MAC, which practically double their PDR results when employing the studied minimum and maximum duty-cycle ratios. The rest present $100 \%$ PDR in all cases. One may observe that B-MAC achieves this in a more linear manner than X-MAC, since as a result of its strobed preamble, the latter shows a slightly better performance in terms of PDR, even at low duty-cycle ratios. However, B-MAC and X-MAC behave in a drastically different way in terms of power consumption. While X-MAC, as might be expected, slightly increases the average nodes' power consumption as the duty-cycle ratio (Fig. 5b), B-MAC decreases its power consumption due to a higher PDR. BMAC's preambles become shorter with higher duty-cycles, effectively reducing the contention. X-MAC collapses for $10 \%$ duty-cycle, since no improvement is observed in terms of PDR

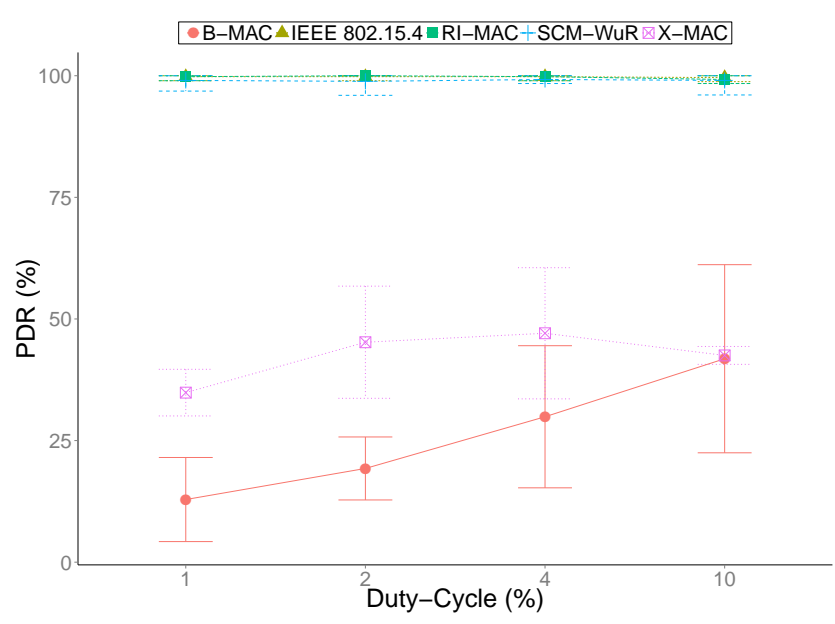

(a)

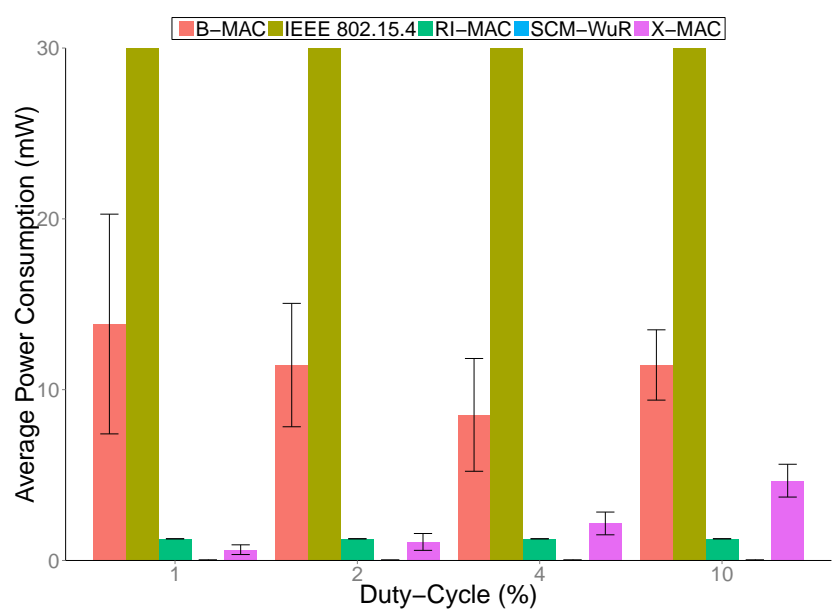

(b)

Fig. 5: The effect of the duty-cycle ratio on (a) the network's PDR and (b) the overall average power consumed by deployed sensor nodes for the single-hop scenario in Fig. 4a.

even if more power is required on average. On the other hand, by presenting no duty-cycle, IEEE 802.15 .4 's power consumption is constant at around $60 \mathrm{~mW}=3 \mathrm{~V} * 17.4 \mathrm{~mA}$ throughout all evaluated results. By considering altogether the graphs in Fig. 5, it is clear that SCM-WuR provides the best trade-off between PDR and consumed power among all approaches, since it only employs $30 \mu \mathrm{W}$ on average.

2) Effect of Coexistent Network Interference: We model the effect of interferers on the network performance by varying the number of dark nodes in Fig. 4a, which generate and transmit packets with a uniform( $1 \mathrm{~s}, 10 \mathrm{~s})$ packet interarrival time distribution. These packets are not intended for any node in the evaluated network, but generate contention as they occupy the wireless medium. The duty-cycle of the MAC approaches is set to $1 \%$ in this evaluation.

Again, we define the PDR as the ratio of responses received from nodes at the mobile node, to the number of performed queries. For protocols based on preambles, the effect of the node contention over the network's PDR may be observed easily in Fig. 6. If a node is placed among several contenders, 
its response may not find its way back to the collector node in time, even after several retransmissions. Because of its strobed preamble, X-MAC performs better than B-MAC under contention, even if both only achieve 100\% PDR for scenarios without any contention. When 30 interferers are present, as evaluated in Section IV-B1, PDR results are analogous, which reaffirms the consistency of the simulations. In turn, IEEE 802.15.4, RI-MAC and SCM-WuR, because of a better management of the wireless medium, provide a PDR very close to $100 \%$ for the considered numbers of interferer nodes.

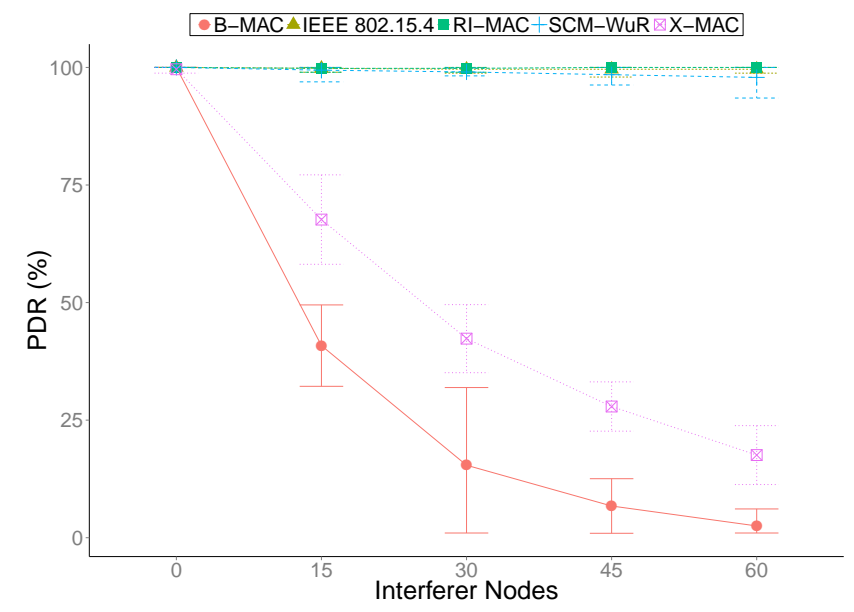

Fig. 6: Effect of the number of interferer nodes on PDR for the single-hop scenario.

Once again, for battery-limited networks such as WSN, PDR results must be assessed together with the power consumed by the network nodes. In Fig. 7, we depict the average lifetime of the network, understood as the average number of days a sensor node in the network shown in Fig. 4a may last without requiring a battery replacement. Lifetime evaluations in Fig. 7 show that, when some degree of interference is expected, B-MAC performs unsatisfactorily in terms of lifetime. The figure shows how WuR extends lifetime by one order of magnitude compared to the duty-cycled approaches. Due to its long preamble, B-MAC shows high power consumption. In turn, X-MAC's strobed preamble allows at least for extended lifetime by detecting unintended preambles from interferer nodes. However, as observed from Fig. 6, these interferers do indeed negatively affect X-MAC's PDR. On the other hand, IEEE 802.15.4 achieves perfect PDR at the cost of the worst lifetime results. RI-MAC, in turn, appears to be the best MAC protocol approach in terms of PDR, mainly because of its clever management of the wireless medium. However, since it is based on duty-cycle, RI-MAC cannot improve the mAlevel current consumption. SCM-WuR also performs the best in this scenario, and shows an optimum performance tradeoff with both excellent PDR and lifetime results thanks to its implicit resilience to $\mathrm{WuC}$ from interferer nodes, which is enabled by the use of hardware address correlation, and its $\mu \mathrm{A}$ level current consumption when in sleep mode, respectively. In fact, WuR is only surpassed in terms of PDR by IEEE 802.15.4. However, this approach is simply not affordable from the energy point of view in this single-hop scenario.

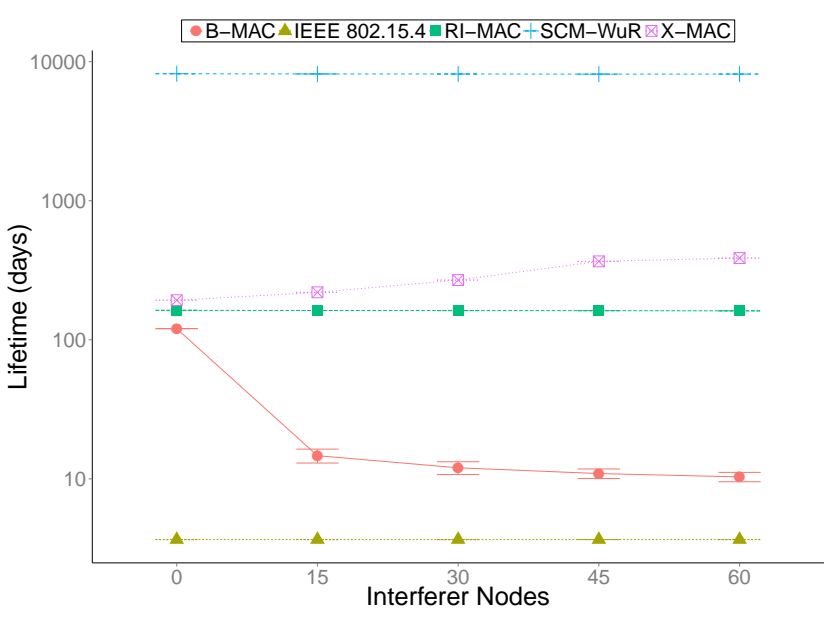

Fig. 7: Effect of the number of interferer nodes on the network lifetime for the single-hop scenario (logarithmic y-axis).

\section{Multi-hop Static Scenario}

In order to effectively compare the performance of the studied approaches for multi-hop static scenarios, we define a binary tree as shown in Fig. 4b, where nodes send packets in a regular manner towards the sink in a multi-hop fashion. Thus, unlike the single-hop scenario in Section IV-B, this scenario follows a Transmitter Initiated paradigm where nodes constantly generate packets instead of waiting to be queried. For the WuR approach, TI-WuR flavour is considered, since the node sending the $\mathrm{WuC}$ transmits a data packet afterwards. Packet routes are predefined to carry out the performance comparison independently of the employed routing protocol. Under this topology, the network nodes periodically generate data packets and send them to their parent nodes, which are in charge of forwarding the packets towards the sink. For this convergecast scenario, we define the PDR to be the number of packets received at the sink over the total number of packets generated during the experiment by all participant nodes in the network. Transmissions from interferer nodes are not taken into account in PDR calculations. No data aggregation strategy is considered.

1) Effect of the Duty-Cycle Ratio: We set the network's nodes to generate a packet every 10 seconds. None of the approaches achieves a $100 \%$ PDR value in this multi-hop static scenario, even for no-contention circumstances, as seen in Fig. 8a. Furthermore, unlike in Section IV-B1, in this case RI-MAC performs as poorly as the other duty-cycled MACs. In fact, only B-MAC achieves a better performance as the duty-cycle increases. Because of its medium-blocking preamble, any increase in the duty-cycle does result in higher transmission probability. On the other hand, an increase in the duty-cycle does not result in better PDR in the case of XMAC and RI-MAC, since with these protocols the network nodes cannot transmit once they detect surrounding strobed preambles or beacons, respectively. Moreover, a node is also unable to process any other communication when performing a retransmission of one packet. Both of these issues result in poor PDR results. On the other hand, in applications where 
transmission depends on a query, such as the single-hop scenario, the benefit of duty-cycle is more evident since nodes do not suffer from pending retransmissions.

For the evaluated packet generation period of 10 seconds, Node 1, as one of the two most energy-demanding nodes in the network in Fig. 4b, is mostly busy. Thus, its power profile does not change significantly when varying duty-cycle ratios for MAC protocols as shown in Fig. 8b.

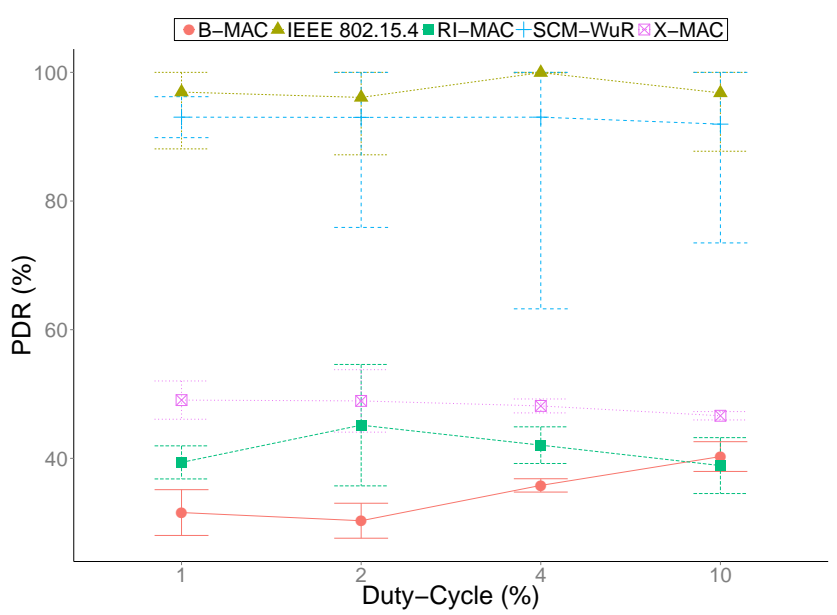

(a)

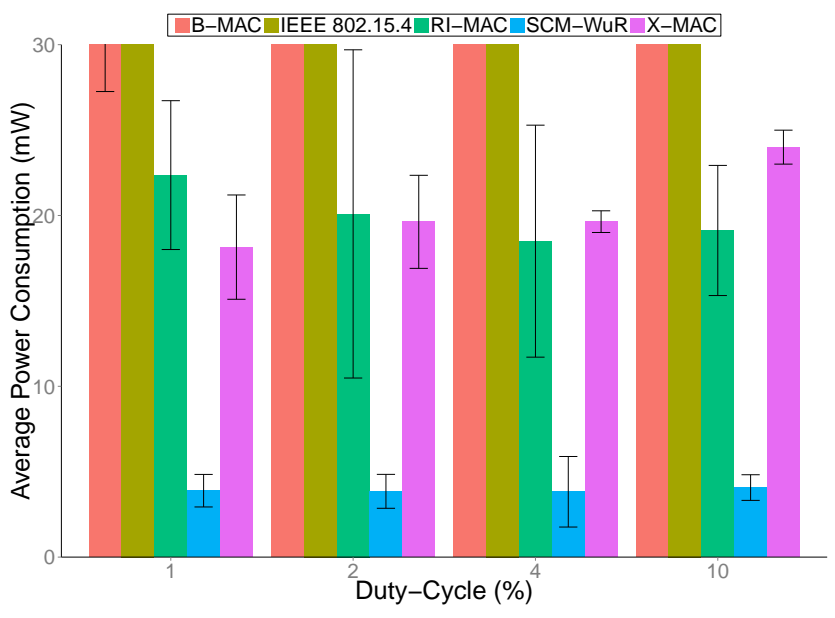

(b)

Fig. 8: Effect of the duty-cycle on (a) the PDR and (b) the average power consumption of Node 1 for the multi-hop static scenario.

The average power consumption values of Node 1 are used to calculate the network lifetime, which is measured to be about 3 days, 12 days, 18 days, 20 days and 140 days in average for IEEE 802.15.4, B-MAC, X-MAC, RI-MAC and SCM-WuR, respectively, for the packet generation rate of 1 packet per 10 seconds. These lifetime values are much lower than those for the single-hop scenario due to the constant and relatively heavier traffic load suffered by the nodes in the tree scenario. In the single-hop scenario, nodes are allowed to sleep for much longer periods of time and queried rarely, yielding less average power consumption and longer lifetimes.
2) Effect of the Packet Generation Period: In order to evaluate the network performance under different traffic loads, i.e., data rates, we vary the time period between consecutive packet generations by nodes in the network. As a numerical example, if the packet generation period is 10 seconds, a node placed at penultimate level of the tree will have to forward 2 packets, 1 from each child, as well as to generate its own packet, for a total of 3 packets every 10 seconds. Nodes closer to the sink are naturally in charge of forwarding many more packets than the nodes closer to the leaf nodes. Packet generation periods are tested by starting at $300 \mathrm{~s}$ and going down gradually to $1 \mathrm{~s}$ in order to increase the traffic load.

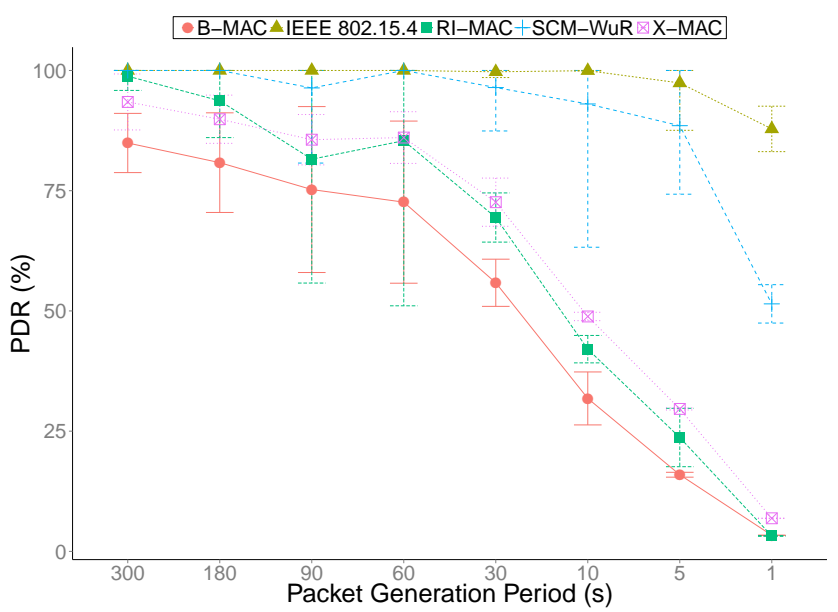

Fig. 9: Effect of the packet generation period on the network PDR for the multi-hop static scenario.

Fig. 9 shows the PDR achieved by the five approaches investigated for different packet traffic loads. As seen in the figure, B-MAC results in a PDR close to $0 \%$ when the time between packet generations is close to 1 second, since the preamble duration is also 1 second, a circumstance which saturates the network. However, few WSN applications are required to transmit this frequently, and packet generation periods longer than 30 seconds are much more common. In B-MAC, wait periods due to busy medium can be significant because of the long preamble duration and, in addition, during this procedure both the transmitting node and the nodes that are detecting the preamble cannot receive any packets from any other node. Moreover, the energy consumption of the surrounding nodes is also increased, since in B-MAC they cannot sleep until they receive the data frame carrying the address of the destination node, which comes after the preamble. Thus, in this topology, the performance of B-MAC quickly collapses for short packet generation periods. For nodes in charge of forwarding packets from a greater number of descendants, this issue is even more pronounced. X-MAC suffers from the same drawback as BMAC, but thanks to its strobed preamble, it slightly diminishes its effect and provides a better PDR. In turn, IEEE 802.15.4 and SCM-WuR provide good PDR values close to $100 \%$, except in the most demanding use case. In the case of WuR, each data communication includes both $\mathrm{WuC}$ and data packet. For packet generation periods of 1 and 5 seconds, Nodes 1 and 2 simply cannot attend all incoming transmissions. On 
the other hand, while in previous scenarios RI-MAC offered a similar performance in terms of average consumed power, but much better PDR than B-MAC and X-MAC, this trend is no longer observed because of the Transmitter Initiated nature of this current multi-hop static scenario.

The mean power profile for the analyzed approaches is shown in Fig. 10a for Node 1 and for Node 11, which has a lighter traffic load, in Fig. 10b. As expected, nodes running IEEE 802.15.4 consume the highest average power among all approaches due to continuous listening of the channel. The purpose of duty-cycled protocols is precisely to reduce such energy-demanding continuous listening. X-MAC and RIMAC effectively accomplish this for packet generation periods larger than 30 seconds. For a packet generation period of 300 seconds, SCM-WuR enables minimum network lifetimes of up to 1000 days of network operation time; RI-MAC and XMAC around 100 days; B-MAC around 65 days, and finally IEEE 802.15 .4 is only able to provide around 3 days. These lifetime values, shown in Fig. 11a, correspond to either Node 1 and/or Node 2, since they are the ones in charge of performing most tasks in the tree shown in Fig. 4b. SCM-WuR clearly outperforms any other approach in an order of magnitude.

Although they may seem to provide similar lifetime results for this scenario, RI-MAC performs worse than X-MAC when taking into account the ratio between total energy featured by the network nodes over the total payload bits received at the sink, as shown in Fig. 11b. Logically, in Fig. 11b, the energy per bit performance of IEEE 802.15.4 improves as the data rate gets higher. The reason for this is that IEEE 802.15.4 consumes energy independently of the traffic rate because of its always-on state; thus, the energy efficiency increases in accordance with the data load. In fact, the energy efficiency of IEEE 802.15.4 is better than that of WuR for very high data rates. However, these rates do not correspond to common WSN use case requirements. Clearly, WuR obtains its energy advantages from the fact that the main radio interface is in the sleep mode for most of the time. Given the long preamble or receiving time they require for each data packet, B-MAC, XMAC and RI-MAC consume more energy per bit than WuR but still less than IEEE 802.15.4 for low traffic loads. The energy per bit trends for these three MAC protocols are similar when the data load is increased.

To assess the delay overhead incurred by $\mathrm{WuR}$, we also evaluated the average latency of each protocol. In this multihop static scenario, the latency of the network is defined as the difference between the time a packet is generated and the packet's reception time at the destination. In Fig. 12, the average latency for a packet to travel from the leaf Node 7 in Fig. 4b to the network's sink is shown as an illustrative example. Such a measure can be seen as the total time for a packet generated at the furthest tree level to go through all the levels of the network's topology. For the preamble-based MACs, a preamble is generated at the same time as a data packet and sent just before it. In B-MAC, data packets may undergo long waiting periods before being transmitted, due to surrounding preamble transmissions. This delay is repeated in all hops up to the sink, and becomes increasingly greater as the data load increases. Once again, MAC approaches saturate for

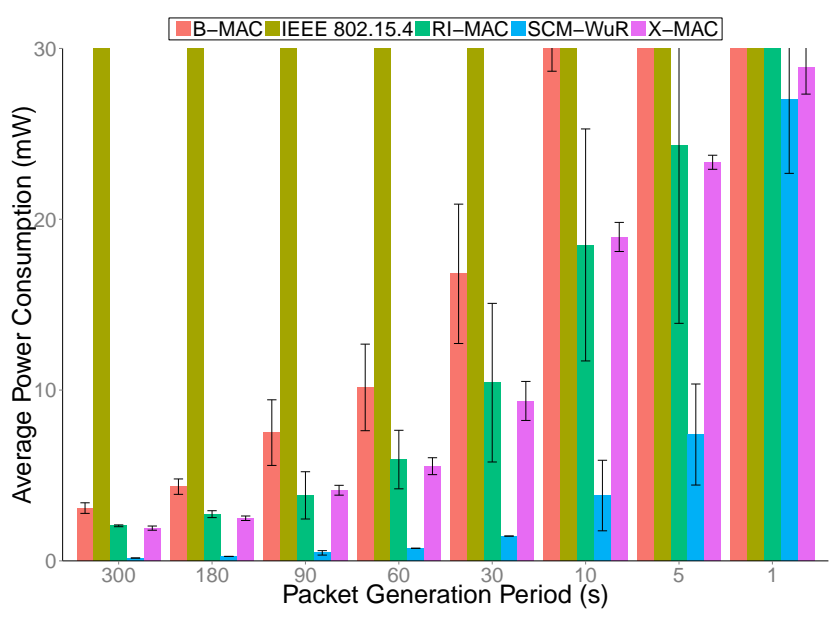

(a)

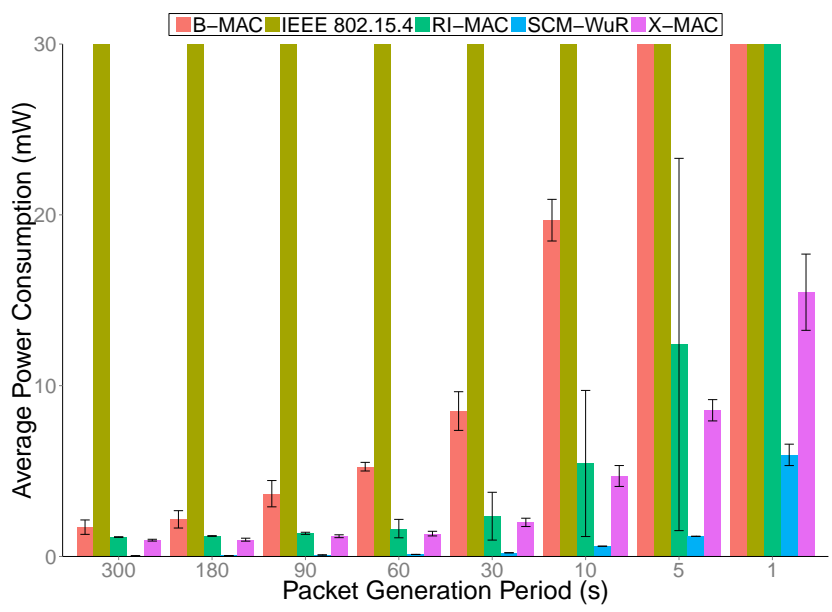

(b)

Fig. 10: Effect of the packet generation period on the average power consumption of (a) Node 1 and (b) Node 11, for the multi-hop static scenario depicted in Fig. 4b.

high data generation rates. On the other hand, IEEE 802.15.4 obtains the best latency results due to being constantly active for monitoring the channel and for not incurring any delay overhead except the CCA prior to the packet transmissions. However, this comes at the cost of a high power consumption. For its part, SCM-WuR performs efficiently in terms of latency when compared to the other two approaches, with values not higher than $60 \mathrm{~ms}$ for packets to go through the entire network. This value corresponds to the summation of the amount of time needed for the $\mathrm{WuC}(12.2 \mathrm{~ms}$ in Table 1); the transition of the MCU and main network interface card from sleep to receiving state $(1.79 \mathrm{~ms})$; the reception of the data packet (time for a 100-byte packet at $250 \mathrm{kbps}$ is $3.2 \mathrm{~ms}$ ), and the average contention and processing-related times $(1.5 \mathrm{~ms})$ for a total of $18.5 \mathrm{~ms}$. This value, multiplied by 3 hops (4 levels) from Node 7 to reach the sink, adds up to a total of approximately $60 \mathrm{~ms}$, which may vary slightly depending on the conditions of contention, collisions, etc. suffered by each communication hop. 


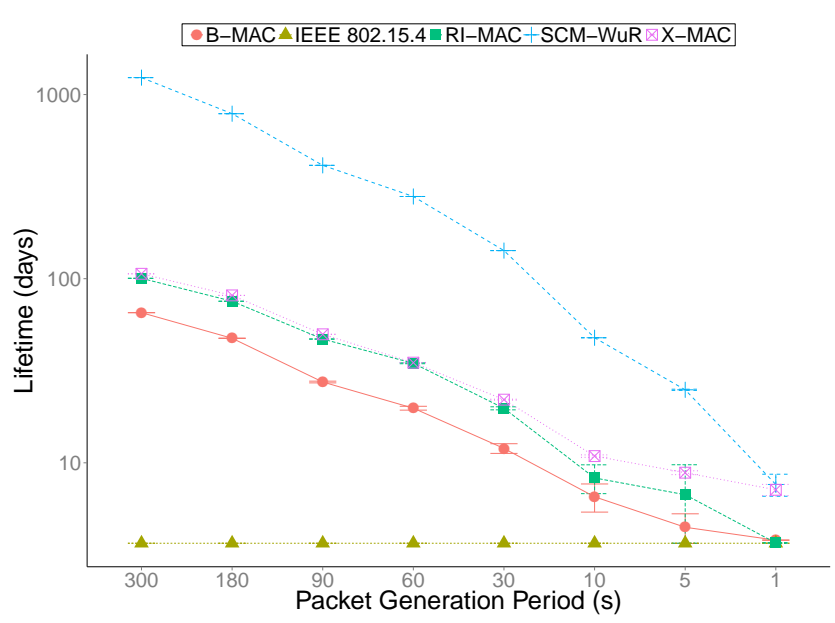

(a)

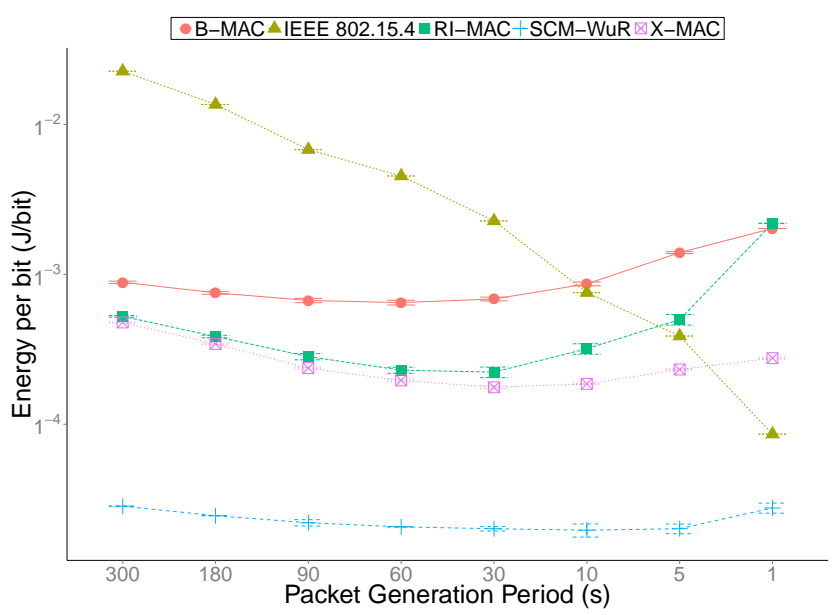

(b)

Fig. 11: Effect of the packet generation period on (a) the network lifetime (logarithmic y-axis) and (b) the energy per received bit for the multi-hop static scenario.

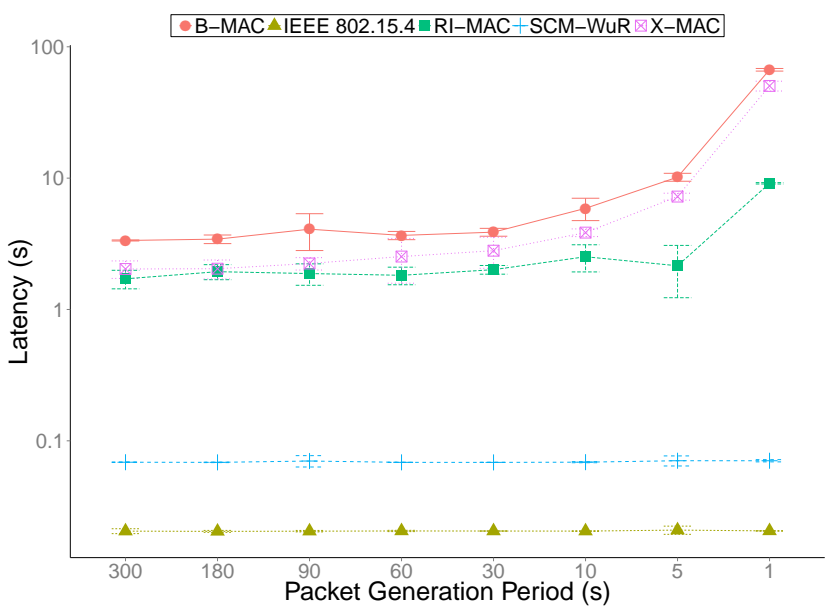

Fig. 12: Effect of the packet generation period on the average latency of Node 7 packets for the multi-hop static scenario (logarithmic y-axis).
3) Effect of Coexistent Network Interference: To isolate and evaluate the effect of the number of interferer nodes, we keep the number of seconds between packet generations to 90. As shown in Fig. 13a, because of the multi-hop nature of the tree scenario, even SCM-WuR and RI-MAC suffer from the effect of interferer nodes and can no longer provide PDR close to $100 \%$ any longer with the 2 retransmissions allowed. Regarding B-MAC and X-MAC, they present the same poor lifetime performance as in the single-hop scenario, as shown in Fig. 13b.

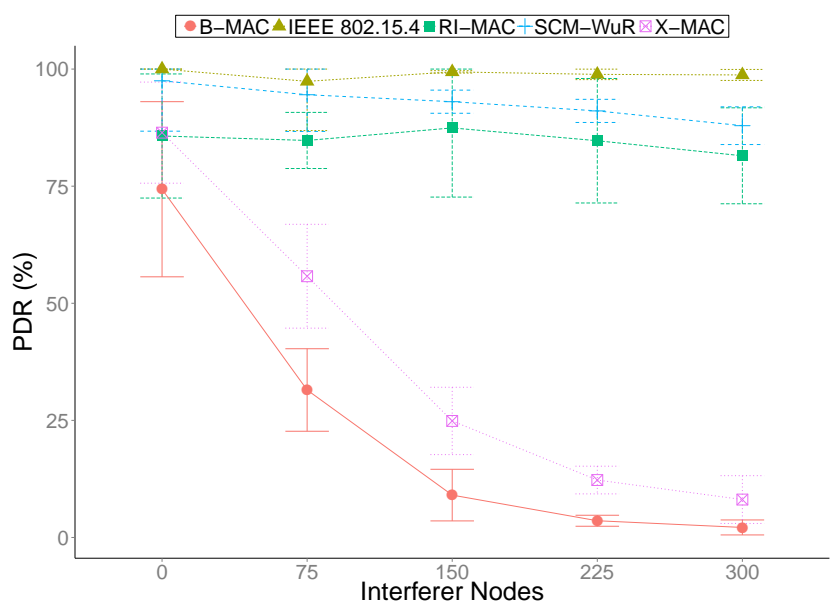

(a)

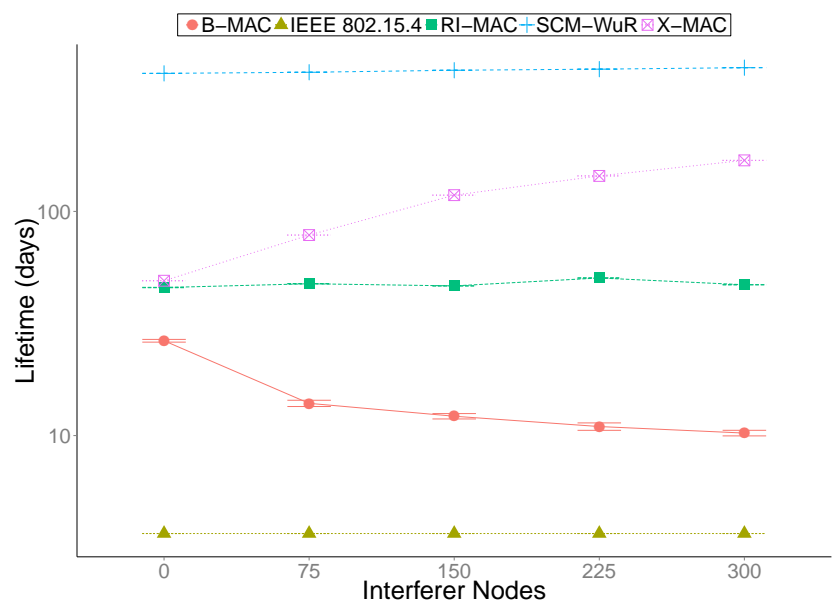

(b)

Fig. 13: Effect of the number of interferer nodes on (a) the network's PDR and (b) the maximum lifetime for the multihop static scenario (logarithmic y-axis).

Once again, in this scenario WuR is the approach offering the best PDR-lifetime trade-off. Only for very high data rates, which in turn are not typical in WSN, IEEE 802.15.4 achieves a good trade-off between power and PDR and emerges as a better candidate due to its lower latency and consequent capability of managing higher data rates. Interestingly, none of the remaining approaches, based on duty-cycling, appear as suitable candidates for this scenario. 


\section{Multi-hop Mobile Scenario}

In the final application, nodes remain idle until the presence of a mobile data-collector, which may be a bus, a car or even a drone, on a bridge. As depicted in Fig. 4c, this mobile node queries the first node in a chain of four nodes placed along the length of bridge's pillars. As in the single-hop scenario case, this is an example of a Receiver Initiated communication. Accordingly, the RI-WuR variant is considered for the WuR approach, i.e., throughout the entire multi-hop communication, a node first sends a $\mathrm{WuC}$ and immediately switches its main radio to reception mode to receive back a data frame. However, as in the tree application, this RI scenario requires multihop communication. For this bridge monitoring application, we program the mobile node to continuously travel back and forth on the bridge or target area. The query travels from the mobile node down to the last node of the pillar and then returns back to the mobile node in a multi-hop fashion. Thus, to recover the information from a bridge's pillar, we perform 8 communication hops, 4 in each direction. It is clear to see that this scenario combines the nature of the previous two. It is for this reason, and because any but the fastest packet rate can be used, that in this section we study the effect of the interferences, the effect of the duty-cycle and the effect of the speed of the mobile node. In regard to the communication delay at each hop, situations may arise where the answer from the bridge's pillar is not detected by the mobile node because it is already out of range as a result of its traveling speed.

1) Effect of the Duty-Cycle Ratio: As in the tree example, in this application an increase of the duty-cycle does not signify an immediate performance improvement. In fact, since the application requires a quick response, B-MAC cannot achieve this by increasing the duty-cycle due to its long preamble. We set the number of interferers to 0 for the duty-cycle evaluation. Otherwise, as we previously observed, in this scenario B-MAC and X-MAC cannot complete any data transaction, not even for the default mobile node's speed of $10 \mathrm{~m} / \mathrm{s}(36 \mathrm{~km} / \mathrm{h})$. The effects of the variation of the duty-cycle can be observed in Fig. 14a and Fig. 14b for network's PDR and latency, respectively. Only if all the nodes in a chain can provide their results, can we regard the entire query as successful for the application PDR.

In Fig. 14a, one may observe that by increasing the dutycycle the PDR for X-MAC improves up to $100 \%$. This behavior is consistent with that analogous for 0 interferers in Fig. 6. In terms of latency, IEEE 802.15.4 performs the full up-down-up communication in $50 \mathrm{~ms}$, while SCM-WuR, RIMAC, X-MAC and B-MAC require $160 \mathrm{~ms}, 4000 \mathrm{~ms}, 4500 \mathrm{~ms}$ and $7500 \mathrm{~ms}$, respectively. Communication is possible as long as the mobile node does not move out of communication range during this time, as analyzed in the following section. On average, B-MAC requires 1 second to communicate at each hop, while RI-MAC and X-MAC require half this time. Thus, the multi-hop latency values can be approximated to the singlehop latency for each approach multiplied by the number of hops.

Regarding battery lifetime, and for a duty-cycle of $2 \%$ for MAC protocols, IEEE 802.15.4, B-MAC, X-MAC, RI-MAC

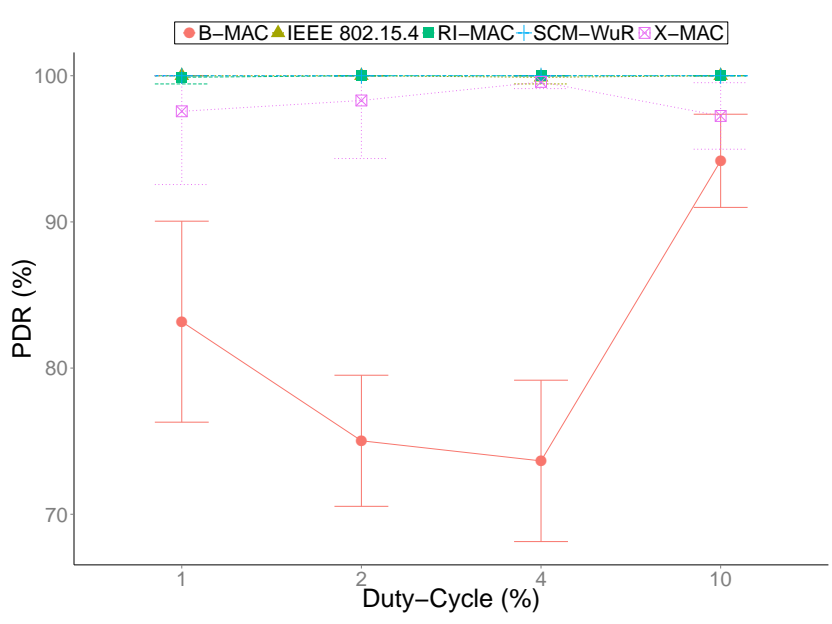

(a)

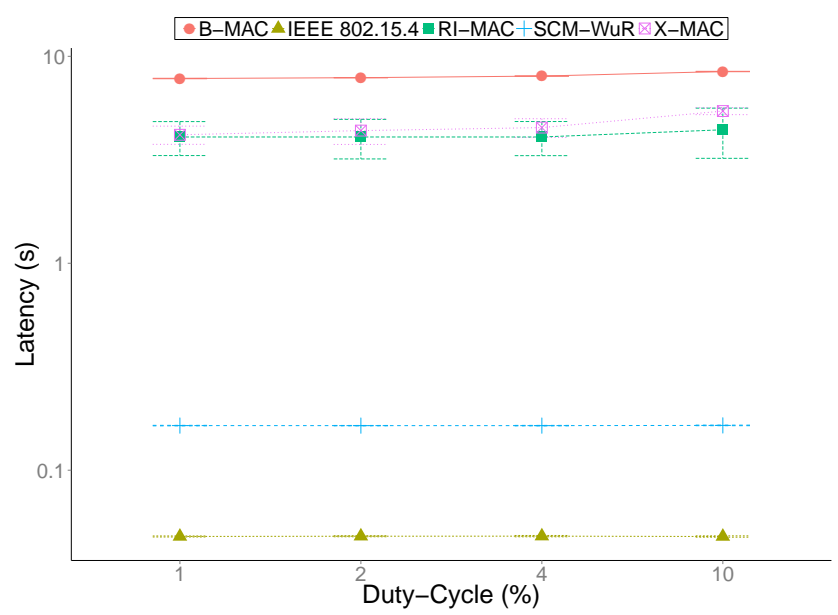

(b)

Fig. 14: Effect of the duty-cycle on (a) the PDR and (b) the latency for the bridge monitoring application in Fig. 4c (logarithmic y-axis).

and SCM-WuR are found to provide respective average node lifetimes of 3 days, 38 days, 82 days, 119 days and 1300 days, respectively. This improvement in orders of magnitude by WuR can also be observed for the other duty-cycle ratios of the MAC approaches evaluated.

2) Effect of Coexistent Network Interference: Unlike the tree application, the traffic generated by the current scenario is not constant. Once the first node in the bridge's pillar is queried, communication must take place quickly in order to recover information from all the nodes in the chain. PDR and energy per bit results are shown in Fig. 15a and 15b respectively, from which it is easy to conclude that Transmitter Initiated MACs, such as B-MAC and X-MAC, do not fit to this type of application very well if contention is present. Failure to deliver the requested information for B-MAC and XMAC causes their energy per bit ratio to noticeably increase. For the other approaches, only slight PDR decreases and energy increments occur as the number of interferer nodes is increased.

In terms of lifetime, SCM-WuR also outstrips the other 


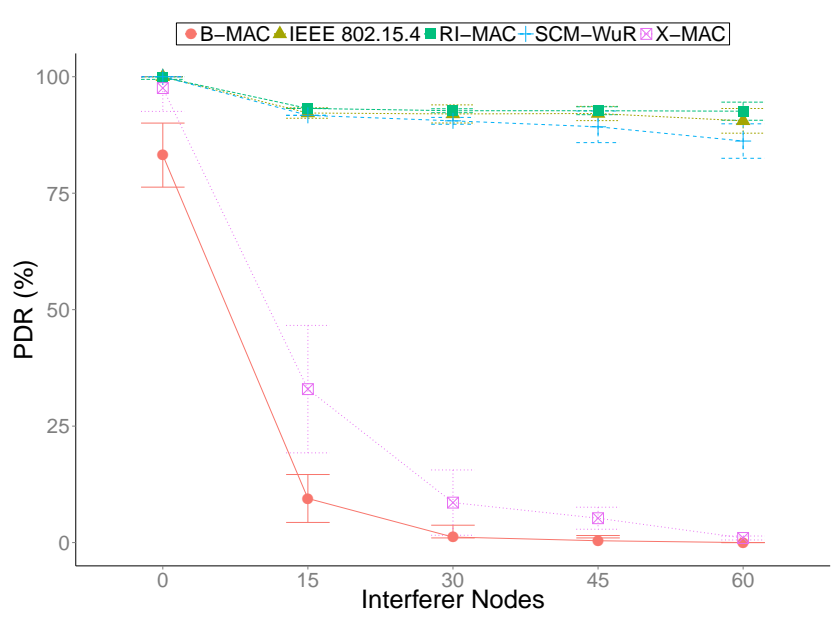

(a)

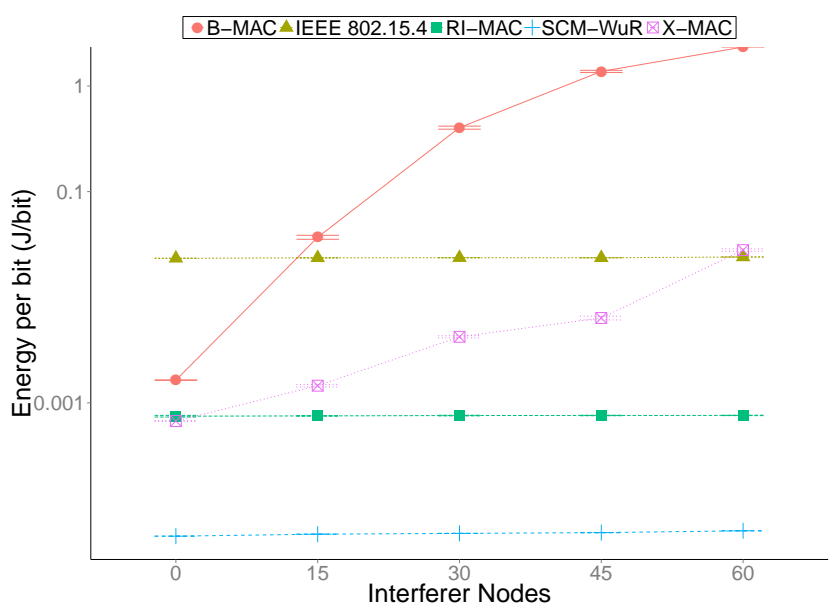

(b)

Fig. 15: Effect of the number of interferer nodes on (a) the PDR and (b) the energy/bit calculation for the bridge monitoring application (logarithmic y-axis).

approaches by a factor of 10 , with up to 1000 days of battery life (not shown in the figures).

3) Effect of the Mobile Node's Speed: Finally, we vary the mobile node's speed in order to determine how fast it can travel and still recover the information from the bridge. Again, we set interferers to zero in order to be able to retrieve information from B-MAC and X-MAC.

As shown in Fig. 16a, at around $25 \mathrm{~m} / \mathrm{s}$ not all the packets are received back by the mobile node for any of the three duty-cycled MAC protocols, which simply cannot offer a good trade-off between PDR, latency and power consumption. This speed limit further decreases in the presence of interferences, as seen in previous sections. For this reason it can be stated that duty-cycled MAC approaches cannot be deployed in applications involving fast multi-hop to a mobile sink. Again, IEEE 802.15.4 provides unbeatable PDR, but only when the most power among all MAC protocol candidates is employed. In turn, SCM-WuR performs best for all mobile speeds investigated in terms of the energy and PDR metrics trade-off.

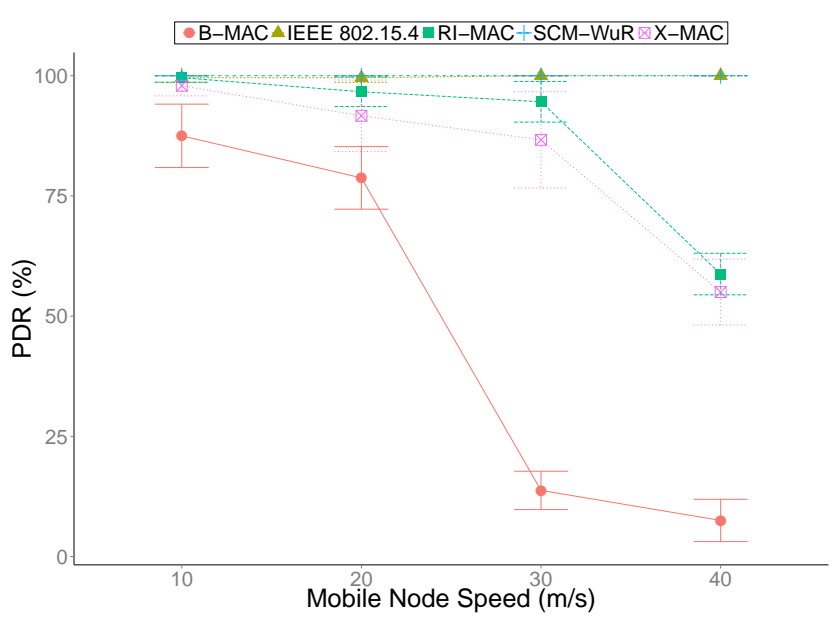

(a)

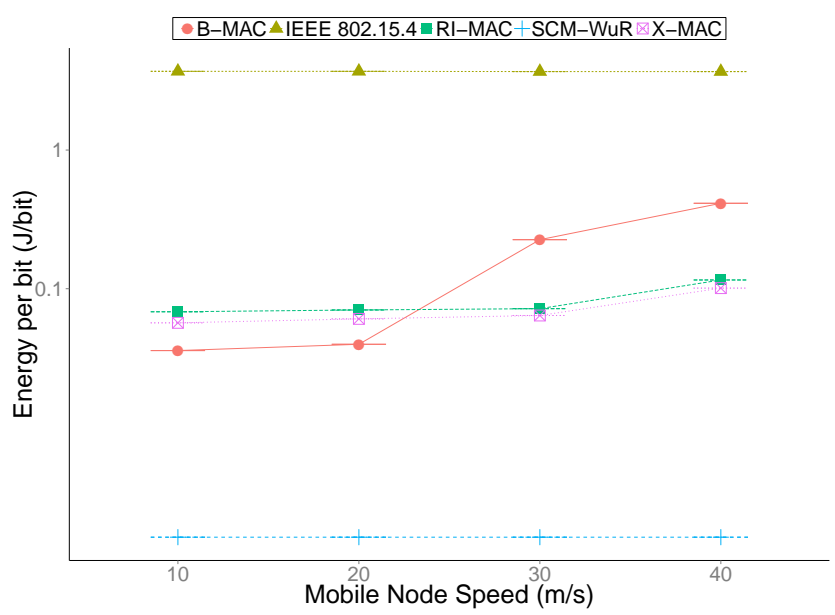

(b)

Fig. 16: Effect of the mobile node's speed on (a) the PDR and (b) the energy per bit for the bridge monitoring application (logarithmic y-axis).

\section{CONCLUSions}

Even if duty-cycled approaches help to reduce current consumption due to idle listening and overhearing, this reduction is insufficient for new low-power designs demands, where nodes should save as much energy as possible providing that their intervention is not required. The use of Wakeup Radio (WuR) provides significant improvements to the current WSN, which are commonly based on duty-cycling MAC strategies. This paper contains numerous OMNET++ simulations for realistic use cases implemented by 4 different duty-cycled MAC protocols and one real WuR platform, called SCM-WuR. Throughout evaluations of these use cases for numerous metrics, it is clearly observable that SCM-WuR constantly allows for substantial energy savings, higher PDR, lower latency and less complicated software implementations compared to duty-cycled MAC protocols. In fact, the only disadvantage of WuR systems appears to be the need for extra hardware development. The results presented in this paper furnish network designers with the fundamentals for seriously considering switching from currently dominant duty-cycled 
networks to a WuR approach, as the authors have already done for several projects deployed around Europe.

\section{REFERENCES}

[1] K. Langendoen, "The MAC Alphabet Soup." [Online]. Available: http://www.st.ewi.tudelft.nl/ koen/MACsoup/protocols.php

[2] V. András and H. Rudolf, "An overview of the OMNeT++ simulation environment," in Proceedings of the 1st international conference on Simulation tools and techniques for communications, networks and systems \& workshops (Simutools). ICST, Brussels, Belgium, Belgium: ICST (Institute for Computer Sciences, Social-Informatics and Telecommunications Engineering), 2008, pp. 1-10.

[3] A. Köpke, M. Swigulski, K. Wessel, D. Willkomm, P. T. K. Haneveld, T. E. V. Parker, O. W. Visser, H. S. Lichte, and S. Valentin, "Simulating wireless and mobile networks in OMNeT++ the MiXiM vision," in Proceedings of the 1st International Conference on Simulation Tools and Techniques for Communications, Networks and Systems \& Workshops (Simutools), Mar. 2008, p. 71.

[4] M. Buettner, G. V. Yee, E. Anderson, and R. Han, "X-MAC: a short preamble MAC protocol for duty-cycled wireless sensor networks," in Proceedings of the 4th international conference on Embedded networked sensor systems. ACM, 2006, pp. 307-320.

[5] Y. Sun, O. Gurewitz, and D. B. Johnson, "RI-MAC: a receiver-initiated asynchronous duty cycle MAC protocol for dynamic traffic loads in wireless sensor networks," in Proceedings of the 6th ACM conference on Embedded network sensor systems (SenSys). ACM Press, Nov. 2008, p. 1.

[6] G. U. Gamm, M. Sippel, M. Kostic, and L. M. Reindl, "Low power wake-up receiver for wireless sensor nodes," in 6th International Conference on Intelligent Sensors, Sensor Networks and Information Processing (ISSNIP). IEEE, 2010, pp. 121-126.

[7] J. Oller, I. Demirkol, J. Casademont, J. Paradells, G. U. Gamm, and L. Reindl, "Performance Evaluation and Comparative Analysis of SubCarrier Modulation Wake-up Radio Systems for Energy-Efficient Wireless Sensor Networks," Sensors, vol. 14, no. 1, pp. 22-51, Dec. 2013.

[8] V. Jelicic, M. Magno, D. Brunelli, V. Bilas, and L. Benini, "Analytic comparison of wake-up receivers for WSNs and benefits over the wake-on radio scheme," in Proceedings of the 7th ACM workshop on Performance monitoring and measurement of heterogeneous wireless and wired networks (PM2HW2N '12). ACM, 2012, pp. 99-106.

[9] R. Su, T. Watteyne, and K. S. J. Pister, "Comparison between Preamble Sampling and Wake-Up Receivers in Wireless Sensor Networks," in IEEE Global Telecommunications Conference (GLOBECOM), 2010, pp. $1-5$.

[10] Y. Zhang and G. Dolmans, "Wake-up radio assisted energy-aware multi-hop relaying for low power communications," in IEEE Wireless Communications and Networking Conference (WCNC). IEEE, Apr. 2012, pp. 2498-2503.

[11] R. Jurdak, A. G. Ruzzelli, and G. M. P. O'Hare, "Adaptive Radio Modes in Sensor Networks: How Deep to Sleep?" in 5th Annual IEEE Communications Society Conference on Sensor, Mesh and Ad Hoc Communications and Networks. IEEE, Jun. 2008, pp. 386-394.

[12] _ - "Radio Sleep Mode Optimization in Wireless Sensor Networks," IEEE Transactions on Mobile Computing, pp. 955-968, 2010.

[13] J. Oller, I. Demirkol, J. Casademont, J. Paradells, G. U. Gamm, and L. Reindl, "Wake-up Radio As an Energy-efficient Alternative to Conventional Wireless Sensor Networks MAC Protocols," in Proceedings of the 16th ACM International Conference on Modeling, Analysis Simulation of Wireless and Mobile Systems, ser. MSWiM '13. New York, NY, USA: ACM, 2013, pp. 173-180. [Online]. Available: http://doi.acm.org/10.1145/2507924.2507955

[14] D. Gislason, ZigBee Wireless Networking. Newnes, 2004.

[15] Z. Shelby and C. Bormann, 6LoWPAN: The Wireless Embedded Internet. Wiley Subscription Services, 2009.

[16] J. Polastre, J. Hill, and D. Culler, "Versatile low power media access for wireless sensor networks," in Proceedings of the 2nd international conference on Embedded networked sensor systems. ACM, 2004, pp. 95-107.

[17] P. Levis, S. Madden, J. Polastre, R. Szewczyk, A. Woo, D. Gay, J. Hill, M. Welsh, E. Brewer, and D. Culler, "TinyOS: An operating system for sensor networks," in Ambient Intelligence. Springer Verlag, 2004.
[18] A. Dunkels, B. Gronvall, and T. Voigt, "Contiki - A Lightweight and Flexible Operating System for Tiny Networked Sensors," in Proceedings of the 29th Annual IEEE International Conference on Local Computer Networks, ser. LCN '04. Washington, DC, USA: IEEE Computer Society, 2004, pp. 455-462.

[19] Texas Instruments, "CC1101: Low-Power Sub-1 GHz RF Transceiver Datasheet," 2012. [Online]. Available: http://www.ti.com/lit/ds/symlink/cc1101.pdf

[20] G. U. Gamm and L. M. Reindl, "Range extension for wireless wake-up receivers," in 9th International Multi-Conference on Systems, Signals and Devices (SSD), 2012, pp. 1-4.

[21] I. Demirkol, J. Paradells, J. Oller, J. Casademont, and A. Calveras, "Energy Efficient Wireless Networking of Sensor Nodes," in $A M A$ Conferences, 2013, pp. 452-456.

[22] ITC Engineering, "Research project IB-ISEB, Intelligent bridges - information system for structure monitoring and maintenance," 2014. [Online]. Available: http://www.itc-engineering.de/index.php?id=195\&L=1

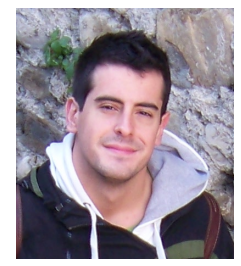

Joaquim Oller did his diploma in Computer Science at the Universitat de Girona, Catalunya. Nowadays he is pursuing his $\mathrm{PhD}$ in the field of wake-up receivers in Telematics Engineering at Universitat Politècnica de Catalunya. He is also concerned with different wireless technologies such as RFID, IEEE802.15.4 and Bluetooth.

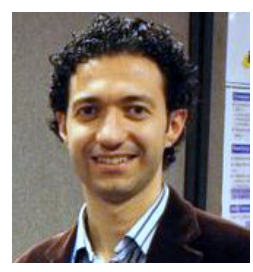

Ilker Demirkol is Postdoctoral Research Associate in Telematics Engineering at Universitat Politècnica de Catalunya, where he works on wireless networks, including wireless mesh, ad hoc and sensor networks, along with wake-up radio systems. Demirkol received his B.Sc., M.Sc., and Ph.D. degrees in Computer Engineering from the Bogazici University, Istanbul, Turkey.

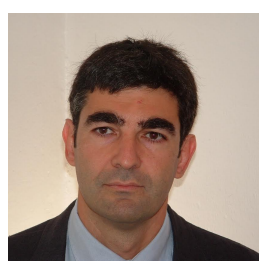

Jordi Casademont is an Associate Professor at the Universitat Politècnica de Catalunya (UPC) in Barcelona. He received his M.S. Degree in Telecommunications in 1992 and the Ph.D. Degree in 1998 $\mathrm{He}$ is an active member of the Wireless Networks Group (WNG) and has published papers in the fields of networking and MAC mechanisms, mesh networks and wireless sensor networks.

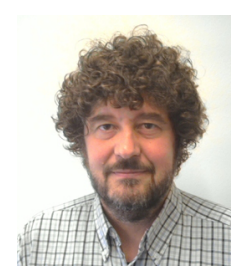

Josep Paradells is the responsible of the Wireless Networks Group (WNG) and the head of the Ubiquitous Internet Technologies Unit (UITU) of the I2Cat Foundation. His expertise areas are network convergence and ambient intelligence, combining theoretical studies with real implementations. He has been participating in national and European publicfunded research projects and collaborating with main Spanish telecommunications companies.

Gerd Ulrich Gamm did his diploma in Electri-

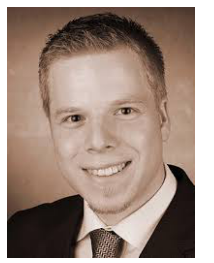
cal Engineering and Information Technology at the Karlsruhe Institute of Technology (KIT), Karlsruhe, Germany. He has recently obtained his $\mathrm{PhD}$ in Microsystems Engineering at the Institute of Microsystems Technology (IMTEK) in Freiburg, Germany. His work is concerned with wake-up receivers for embedded microsystems.

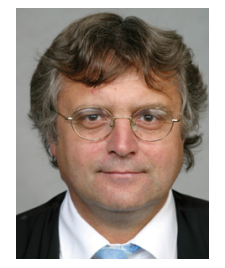

Leonhard Reindl is Head of the Laboratory for Electrical Instrumentation at the Department of Microsystems Engineering, of the University of Freiburg since May 2003. He received a Dipl. Phys. degree from the Technical University of Munich, Germany, in 1985 and a Dr. Sc. Techn. degree from the University of Technology Vienna, Austria, in 1997. His research interests include wireless sensor and identification systems, and surface acoustic wave devices. 